\title{
INTERPOLATION OF WEIGHTED AND VECTOR-VALUED HARDY SPACES
}

\author{
SERGUEI V. KISLIAKOV AND QUANHUA XU
}

\begin{abstract}
Real and complex interpolation methods, when applied to the couple $\left(H^{p_{0}}\left(E_{0} ; w_{0}\right), H^{p_{1}}\left(E_{1} ; w_{1}\right)\right)$, give what is expected if $E_{0}$ and $E_{1}$ are quasi-Banach lattices of measurable functions satisfying certain mild conditions and if $\log \left(w_{0}^{1 / p_{0}} w_{1}^{-1 / p_{1}}\right) \in \mathrm{BMO}\left(w_{0}, w_{1}\right.$ being weights on the unit circle). The last condition is in fact necessary. (It is expected, of course, that the resulting spaces coincide with the subspaces of analytic functions in the corresponding interpolation spaces for the couple $\left(L^{p_{0}}\left(E_{0} ; w_{0}\right), L^{p_{1}}\left(E_{1} ; w_{1}\right)\right)$.)
\end{abstract}

\section{INTRODUCTION}

Let $H^{p} \quad(0<p \leq \infty)$ be the classical Hardy space of analytic functions in the unit disc of the complex plane. It is well known by now that for $0<p_{0}, p_{1} \leq \infty$ and $0<\theta<1$

$$
\left(H^{p_{0}}, H^{p_{1}}\right)_{\theta p}=H^{p} \quad \text { and } \quad\left(H^{p_{0}}, H^{p_{1}}\right)_{\theta}=H^{p},
$$

where $\frac{1}{p}=\frac{1-\theta}{p_{0}}+\frac{\theta}{p_{1}}$. Here $(\cdot, \cdot)_{\theta p}$ and $(\cdot, \cdot)_{\theta}$ denote respectively the real and complex interpolation spaces. Recall that the $L^{p}$-space version of $(0.1)$ is classical. By using the Riesz projection and the standard factorization of functions in $H^{p}$, one can easily derive $(0.1)$ from this $L^{p}$-space version if $p_{0}$ and $p_{1}$ are finite. In the case where one of the indices $p_{0}$ and $p_{1}$ is infinite $(0.1)$ is much deeper, and was established by Jones about ten years ago (cf. [J]).

In this paper we extend (0.1) in two directions. Our first aim is to examine the weighted version of $(0.1)$. We shall consider weights $w$ on the unit circle T such that $\log w \in L^{1}$. Let then $H^{p}(w)$ be the weighted Hardy space (see the next section for the precise definition). Let $w_{0}, w_{1}$ be two weights, $0<$ $p_{0}, p_{1} \leq \infty$ and $0<\theta<1$. We ask whether the following equalities hold:

$(0.2)\left(H^{p_{0}}\left(w_{0}\right), H^{p_{1}}\left(w_{1}\right)\right)_{\theta p}=H^{p}(w)$ and $\left(H^{p_{0}}\left(w_{0}\right), H^{p_{1}}\left(w_{1}\right)\right)_{\theta}=H^{p}(w)$, where $\frac{1}{p}=\frac{1-\theta}{p_{0}}+\frac{\theta}{p_{1}}$ and $w=w_{0}^{p(1-\theta) / p_{0}} w_{1}^{p \theta / p_{1}}$. Here and below we make the convention that $H^{\infty}(w)=H^{\infty}$. Recall also that the $L^{p}$-space version of $(0.2)$ is well known.

Very recently, Cwikel, McCarthy, and Wolff [CMW] have studied (0.2) in the case where $p_{0}=p_{1}<\infty$. They have proved that then $(0.2)$ is true iff

Received by the editors August 10, 1991.

1991 Mathematics Subject Classification. Primary 46E15, 46E40, 46M35, 42B30.

Key words and phrases. Interpolation, Hardy space, Banach lattice, $A_{p}$-weight, Hilbert transform, BMO. 
$\log \left(w_{0} w_{1}^{-1}\right) \in \mathrm{BMO}$. In this paper, we shall show that in the general case (0.2) holds iff $\log \left(w_{0}^{1 / p_{0}} w_{1}^{-1 / p_{1}}\right) \in \mathrm{BMO}$. We thus extend the above result of [CMW] to all indices. Moreover, if this BMO condition is satisfied, we establish the following sharp result underlying the first equality in (0.2): If $f \in H^{p_{0}}\left(w_{0}\right)+$ $H^{p_{1}}\left(w_{1}\right)$ is represented as $f=f_{0}+f_{1}$ with $f_{j} \in L^{p_{j}}\left(w_{j}\right) \quad(j=0,1)$, then there is an another representation $f=g_{0}+g_{1}$ with $g_{j} \in H^{p_{j}}\left(w_{j}\right) \quad(j=0,1)$ and $\left\|g_{j}\right\|_{H^{p_{j}}\left(w_{j}\right)} \leq C\left\|f_{j}\right\|_{L^{p_{j}}\left(w_{j}\right)}(j=0,1), C$ being a constant independent of $f$. In terms of the $K$-functionals (see $\S 1$ ) this can be restated as follows: For any $t>0$ and any $f \in H^{p_{0}}\left(w_{0}\right)+H^{p_{1}}\left(w_{1}\right)$

$$
K\left(t, f ; H^{p_{0}}\left(w_{0}\right), H^{p_{1}}\left(w_{1}\right)\right) \leq C K\left(t, f ; L^{p_{0}}\left(w_{0}\right), L^{p_{1}}\left(w_{1}\right)\right),
$$

where $C$ is a constant depending on $p_{0}, p_{1}$ and the norm of $\log \left(w_{0}^{1 / p_{0}} w_{1}^{-1 / p_{1}}\right)$ in BMO only.

It is worth noting that results like (0.2) not only are interesting in themselves but also have applications in Analysis. In [CMW], for example, the case $p_{0}=p_{1}$ has been applied to obtain boundedness conditions for Toeplitz operators on some weighted Hardy spaces. Another interesting case is $p_{0}<\infty$ and $p_{1}=\infty$. Then the condition on weights reads as $\log w_{0} \in \mathrm{BMO}$. It is easy to see that for any weight $u \in L^{1}$ one can construct another weight $w_{0} \in L^{1}$ such that $w_{0} \geq u, \log w_{0} \in \mathrm{BMO}, \int w_{0}$ is controlled by $\int u$ and the BMO-norm of $\log w_{0}$ by an absolute constant. By using $(0.2)$ for such $w_{0}$, it is possible to deduce the result of Bourgain [B1] stating that every bounded linear operator from the disc algebra to $L^{1}$ is 2-summing (i.e., the analogue of the famous Grothendieck theorem for the disc algebra holds). See [K1 and K4] for more details.

The second objective of this paper is to study a vector-valued version of $(0.1)$. Given an interpolation couple of (complex) Banach spaces $\left(E_{0}, E_{1}\right)$, we consider the interpolation couple $\left(H^{p_{0}}\left(E_{0}\right), H^{p_{1}}\left(E_{1}\right)\right)$ of Hardy spaces with values in $E_{0}$ and $E_{1}$ respectively (cf. the next section for the definition of these spaces). Do we have

$$
\begin{gathered}
\left.\left(H^{p_{0}}\left(E_{0}\right), H^{p_{1}}\left(E_{1}\right)\right)_{\theta p}=H^{p}\left(\left(E_{0}, E_{1}\right)\right)_{\theta p}\right), \\
\left.\left(H^{p_{0}}\left(E_{0}\right), H^{p_{1}}\left(E_{1}\right)\right)_{\theta}=H^{p}\left(\left(E_{0}, E_{1}\right)\right)_{\theta}\right),
\end{gathered}
$$

with $\frac{1}{p}=\frac{1-\theta}{p_{0}}+\frac{\theta}{p_{1}}$ ? Though the answer is negative in general (cf. [BX]), in many interesting cases it is positive. For example, Bourgain [B1] proved the following: There exists an absolute constant $C>0$ such that for any $t>0$ and $f \in H^{1}\left(l_{n}^{1}\right)+H^{1}\left(l_{n}^{\infty}\right)$

$$
K\left(t, f ; H^{1}\left(l_{n}^{1}\right), H^{1}\left(l_{n}^{\infty}\right)\right) \leq C K\left(t, f ; L^{1}\left(l_{n}^{1}\right), L^{1}\left(l_{n}^{\infty}\right)\right) .
$$

This yields immediately

$$
\left(H^{1}\left(l_{n}^{1}\right), H^{1}\left(l_{n}^{\infty}\right)\right)_{\theta 1}=H^{1}\left(l_{n}^{q 1}\right), \quad \text { uniformly in } n,
$$

where $\frac{1}{q}=1-\theta$ and $l_{n}^{q 1}$ is the Lorentz space on $\{1, \ldots, n\}$. In [B1] one can find some interesting applications of $(0.6)$ to the theory of analytic functions in the unit disc. By using a duality-factorization argument of Haagerup and Pisier [HP], one can easily deduce that $(0.6)$ is still valid if $H^{1}$ is replaced by $H^{p}$ for any $0<p \leq \infty$. The case $p=\infty$ is of special interest because of 
several important applications to $(q, p)$-summing operators on the disc algebra (cf. [K2, K4 and P1]).

In this paper, we show that (0.4) and (0.5) are true for certain Banach (and even quasi-Banach) lattices. The result on the real interpolation reads roughly as follows: Given a couple of quasi-Banach lattices $\left(E_{0}, E_{1}\right)$ of measurable functions satisfying certain (mild) conditions, we have for $\left.0<p_{0}, p_{1}<\infty, t\right\rangle$ 0 and any $f \in H^{p_{0}}\left(E_{0}\right)+H^{p_{1}}\left(E_{1}\right)$

$$
K\left(t, f ; H^{p_{0}}\left(E_{0}\right), H^{p_{1}}\left(E_{1}\right)\right) \leq C K\left(t, f ; L^{p_{0}}\left(E_{0}\right), L^{p_{1}}\left(E_{1}\right)\right),
$$

where $C$ is a constant independent of $t$ and $f$. Recall once more that (0.7) means simply that if $f \in H^{p_{0}}\left(E_{0}\right)+H^{p_{1}}\left(E_{1}\right)$ is decomposed as $f=f_{0}+f_{1}$ with $f_{j} \in L^{p_{j}}\left(E_{j}\right) \quad(j=0,1)$, then one can find another decompositon $f=g_{0}+g_{1}$ where $g_{j} \in H^{p_{j}}\left(E_{j}\right) \quad(j=0,1)$ and the magnitude of the norm of $g_{j}$ in $H^{p_{j}}\left(E_{j}\right)$ is roughly the same as that of $f_{j}$ in $L^{p_{j}}\left(E_{j}\right) \quad(j=0,1)$. We show that $(0.7)$ is also true in many cases for $p_{0}=p_{1}=\infty$. (0.7) implies, of course, $(0.4)$. These results generalize those in [X1]. With the similar conditions on $E_{0}$ and $E_{1}$, we also prove $(0.5)$.

Note that in fact we establish weighted versions of $(0.4)-(0.7)$.

The techniques used in this paper are based on [K1-K4] and partly on [X1$\mathrm{X} 2$ ]. They heavily rely upon standard facts of the theory of analytic functions in the unit disc (such as the outer function construction, factorization) and Fourier analysis (weighted norm inequalities, etc.). The first-named author has used similar techniques to study linear topological properties of spaces of analytic functions, especially, of the disc algebra. In particular, he has found simpler proofs of many results of Bourgain (cf. [B1, B2]), as well as certain new facts on $(q, p)$-summing operators on the disc algebra. The second named author has applied these techniques to some partial cases of the problems considered in the present paper. Though probably somewhat tricky, our methods have the advantage of giving in most cases explicit formulae for the functions desired. Note also that our constructions leading to the decompositions expressed by (0.3) and (0.7) are rather short; on the other hand, the case of the complex interpolation method will require more patience from the reader.

Let us mention the Pisier has also recently been considering the interpolation problem for Hardy spaces. He has elaborated a very elegant method completely different from ours that gives certain of our results (for example, $(0.7)$ for some quasi-Banach lattices, cf. [P2]). His method, however, does not seem to work in the weighted case and also in the case of the complex interpolation for vectorvalued Hardy spaces. It should be noted that Pisier's method is easily extendable to give results like $(0.7)$ if $E_{0}, E_{1}$ are Schatten classes; it leads also to some other noncommutative generalizations.

Finally, we note that one can also consider interpolation problems for Hardy spaces defined by real variable methods (i.e., in terms of maximal functions or harmonic vector fields, etc.). For them the problems in question are of very different nature and the answers are known for the most part. In particular, analogues of (0.1) and its vector-valued versions (0.4) and (0.5) hold. We refer to [FRS] for the real interpolation, to $[\mathrm{JJ}]$ for the complex interpolation and to [BX] for the vector-valued case.

The paper is organized as follows. We present the necessary preliminaries in $\S 1$. In $\S 2$, we prove that the BMO condition mentioned at the beginning is 
necessary for (0.2). The real interpolation results for weighted Hardy spaces of scalar-valued functions are presented in $\S 3$. Section 4 is devoted to the complex interpolation. In $\S \S 5$ and 6 the same is done for weighted Hardy spaces of vector-valued functions. Formally, the material of $\S \S 3$ and 4 is covered by $\S \S 5$ and 6 , but we have decided to present the scalar case separately because of its importance and the fact that the proofs are then slightly less involved. In $\S 7$, we deal with the limit case of $(0.7)$ where $p_{0}=p_{1}=\infty$.

\section{Preliminaries}

Let $D$ be the unit disc of the complex plane, $T$ the unit circle equipped with normalized Lebesgue measure $m$. Given $0<p \leq \infty$, we denote by $H^{p}$ the classical Hardy space of analytic functions in $D$. Identifying functions in $H^{p}$ with their boundary values on $\mathrm{T}$, we may regard $H^{p}$ as a closed subspace of $L^{p}(\mathbf{T} ; m)=L^{p}$.

For $1 \leq p \leq \infty$ we denote by $A_{p}$ the class of all weights on $\mathbf{T}$ satisfying the Muckenhoupt $A_{p}$-condition (cf. [GR, T]). Let $H$ and $M$ be respectively the Hilbert transform and the Hardy-Littlewood maximal operator on $\mathbf{T}$. Recall that $w \in A_{1}$ iff $M w \leq C w$, a.e. on $\mathrm{T}$ for some constant $C$ and that $A_{p} \subset A_{q}$ if $p \leq q$. Recall also that if $w \in A_{p} \quad(1<p<\infty)$, then $H$ and $M$ are bounded operators from $L^{p}(w) \quad\left(=L^{p}(w d m)\right)$ into itself; if $w \in A_{1}$, they are bounded from $L^{1}(w)$ into weak- $L^{1}(w)$. We shall need the following (now classical) characterization of $A_{p}$-weights (cf., e.g., [T and GR]).

Jones' Factorization Theorem. $A$ weight $v$ is in $A_{p} \quad(1<p<\infty)$ if and only if there exist $A_{1}$-weights $v_{0}$ and $v_{1}$ such that $v=v_{0} v_{1}^{1-p}$. Moreover, for each fixed $p$ the $A_{1}$-constants of $v_{0}$ and $v_{1}$ can be estimated in terms of the $A_{p}$ constant of $v$ and vice-versa.

Note that the "if" part is quite easy (cf., e.g., [GR]).

The theorem implies, in particular, that every $A_{2}$-weight $v$ can be written in the form $v=v_{0} v_{1}^{-1}$ with $v_{0}, v_{1} \in A_{1}$. We shall need similar factorizations with richer structure (note that in fact many of them are implicit in [ $T$ and GR]).

Lemma 1.1. Given two $A_{1}$-weights $v_{0}, v_{1}$ and a number $K \geq 2$, one can find two weights $u_{0}, u_{1}$ satisfying $v_{0} v_{1}^{-1}=\left(u_{0} u_{1}^{-1}\right)^{2 K}$ and $\left|H\left(u_{0}\right)\right| \leq C u_{0}, M\left(u_{j}^{2}\right) \leq$ $C u_{j}^{2}(j=0,1)$. Here $C$ depends only on $K$ and the $A_{1}$-constants of $v_{0}$ and $v_{1}$.

Proof. Set $\psi_{0} \equiv 1$ and inductively

$$
\begin{aligned}
\psi_{n}= & v_{0}^{-1 / 2 K}\left|H\left(v_{0}^{1 / 2 K} \psi_{n-1}\right)\right|+v_{0}^{-1 / 2 K}\left(M\left(v_{0}^{1 / K} \psi_{n-1}^{2}\right)\right)^{1 / 2} \\
& +v_{1}^{-1 / 2 K}\left(M\left(v_{1}^{1 / K} \psi_{n-1}^{2}\right)\right)^{1 / 2}
\end{aligned}
$$

Since $v_{j}^{-1} \in A_{2} \subset A_{K} \subset A_{2 K}$, it follows that $M$ is bounded on $L^{\grave{K}}\left(v_{j}^{-1}\right)$ and $H$ on $L^{2 K}\left(v_{0}^{-1}\right)$. Hence there is a constant $C$ such that

$$
\left\|\psi_{n}\right\|_{L^{2 K}} \leq C\left\|\psi_{n-1}\right\|_{L^{2 K}} \leq \cdots \leq C^{n}\left\|\psi_{0}\right\|_{L^{2 K}}=C^{n} .
$$

Therefore, the series $\sum_{n \geq 0}(2 C)^{-n} \psi_{n} \equiv \psi$ converges in $L^{2 K}$ and it is immediate that $\left|H\left(v_{0}^{1 / 2 K} \psi\right)\right| \leq 2 C v_{0}^{1 / 2 K} \psi, M\left(v_{j}^{1 / K} \psi^{2}\right) \leq 4 C^{2} v_{j}^{1 / K} \psi^{2}, j=0,1$. Hence, we can take $u_{j}=v_{j}^{1 / 2 K} \psi(j=0,1)$. 
Remark 1.2. By the construction, $\psi$ is in $L^{2 K}$. Since on the unit circle every $A_{1}$-weight is integrable, we have also that $v_{j}^{1 / 2 K} \in L^{2 K}$. Thus, $u_{j} \in L^{K}$, that will be of some use in the sequel.

In this paper, we shall consider only weights $w$ satisfying $\log w \in L^{1}$. This is true for all nonzero $A_{p}$-weights. If $\log w \in L^{1}$, there exists an outer function $\varphi$ in $D$ with $|\varphi|=w$ a.e. on T. Given $0<p<\infty$, we define the weighted Hardy space $H^{p}(w)$ by

$$
H^{p}(w)=\left\{f: f \varphi^{1 / p} \in H^{p}\right\}
$$

and for $f \in H^{p}(w)$

$$
\|f\|_{H^{p}(w)}=\left\|f \varphi^{1 / p}\right\|_{H^{p}}=\left(\int_{\mathbf{T}}|f|^{p} w d w\right)^{1 / p} .
$$

Clearly, $H^{p}(w)$ is a closed subspace of $L^{p}(w)$. The reader is referred to [G, GR and T] for more information on Hardy spaces.

We now describe vector-valued Hardy spaces. Let $(\Omega, \mu)$ be a measure space. We suppose it is $\sigma$-finite for the sake of simplicity. By a quasi-Banach lattice of measurable functions on $(\Omega, \mu)$ we mean any complete quasi-normed space $(E,\|\cdot\|)$ of $\mu$-measurable functions subject to the following condition: if $f \in E$ and $g$ is measurable such that $|g| \leq|f|$ a.e. on $\Omega$, then $g \in E$ and $\|g\| \leq\|f\|$. Let $0<\alpha<\infty$. Define

$$
E^{(\alpha)}=\left\{x:|x|^{\alpha} \in E\right\} \quad \text { and }\|x\|_{E^{(\alpha)}}=\left\||x|^{\alpha}\right\|_{E}^{1 / \alpha} \text { for } x \in E^{(\alpha)} .
$$

Then $E^{(\alpha)}$ is also a quasi-Banach lattice of measurable functions on $(\Omega, \mu)$. For technical reasons, we shall always assume that for some $\alpha>0 E^{(\alpha)}$ admits an equivalent Banach lattice norm. Since every Banach lattice of measurable functions on a $\sigma$-finite measure space possesses a strictly positive order continuous functional (cf., e.g., [KA, Chapter 4, §1, Theorem 5]), it follows that $E$ embeds into $L^{r}(\Omega, \varphi d \mu)$ for some density $\varphi$ with $\varphi>0$ a.e. on the support of $E$. Replacing $\varphi$ by a smaller function we can assume that $\int \varphi d \mu<\infty$.

Given $0<p \leq \infty$, we define $L^{p}(E)=L^{p}(E ; \mathbf{T})$ as the space of all measurable functions $f$ on $(\mathbf{T} \times \Omega, m \times \mu)$ such that $f(z, \cdot) \in E$ for almost every $z \in \mathbf{T}$ and the function $z \mapsto\|f(z, \cdot)\|_{E}$ is in $L^{p}$. The quasi-norm in this space is given by the expression $\left(\int_{T}\|f(z, \cdot)\|_{E}^{p} d m(z)\right)^{1 / p}$ (with the usual convention for $p=\infty)$. Then $L^{p}(E)$ is a quasi-Banach lattice of measurable functions on $(\mathbf{T} \times \Omega, m \times \mu)$ and it embeds into $L^{s}(\mathbf{T} \times \Omega, m \times \varphi d \mu)$, where $s=\min (p, r)$ and $\varphi$ is the density described above.

By $H^{p}(E)$ we denote the subspace of $L^{p}(E)$ consisting of all the functions $f$ such that for some $0<s \leq \infty, f(\cdot, \omega) \in H^{s}$ for almost every $\omega \in \Omega$. We shall often say in this situation that $f$ is analytic in the first variable. Clearly, we can take $\min (p, r)$ for $s$, and the above embedding allows us to prove that $H^{p}(E)$ is a closed subspace of $L^{p}(E)$.

We remark at once that the above definition of $L^{p}(E)$ is different from the usual one that we are going to describe now. If $X$ is a quasi-Banach space and $0<p \leq \infty$, it is customary to define $L^{p}(X)=L^{p}(X ; \mathbf{T})$ as the space of all strongly measurable $X$-valued functions $f$ on $\mathbf{T}$ such that $\|f\|_{X} \in L^{p} \quad(f$ is said to be strongly measurable if it is a pointwise norm-limit of a sequence of simple functions). For distinguishing this latter space from that defined 
previously, we shall denote it by $\widetilde{L}^{p}(X)$. The corresponding Hardy space is denoted by $\widetilde{H}^{p}(X)$. This is the closure in $\widetilde{L}^{p}(x)$ of all complex polynomials with coefficients in $X$ if $0<p<\infty$, and for $p=\infty$ (if $X$ is a Banach space) this is the closed subspace in $\widetilde{L}^{\infty}(X)$ of all functions whose Fourier coefficients vanish on negative integers. Thus for a quasi-Banach lattice $E$ we have two couples of spaces, $L^{p}(E), H^{p}(E)$ and $\widetilde{L}^{p}(E), \widetilde{H}^{p}(E)$, which do not coincide in general. There are, however, many cases in which $L^{p}(E)=\widetilde{L}^{p}(E)$ and $H^{p}(E)=\widetilde{H}^{p}(E)$. For example, this is true for $0<p<\infty$ if $E$ is a reflexive Banach space, and a fortiori, if $E$ is a UMD space.

Recall that a UMD space is a Banach space $X$ such that the Hilbert transform $H$ induces a bounded operator from $\widetilde{L}^{2}(X)$ into itself. We shall denote this induced operator still by $H$, though probably it would be more rigorous to write $H \otimes i d_{X}$. It is well known that if $X$ is a UMD space then $H$ is also bounded from $\widetilde{L}^{p}(X)$ into itself for every $1<p<\infty$. Recall also that $L^{q}(\Omega, \mu)$ is UMD for $1<q<\infty$. See [B3, Bu] for information on UMD spaces.

Given a quasi-Banach lattice $E$ of measurable functions we shall often need that $E^{(\alpha)}$ be UMD for some $\alpha>0$. By this we mean that $E^{(\alpha)}$ admits an equivalent Banach lattice norm for which $E^{(\alpha)}$ becomes a UMD space. Note that in this case $E^{(\beta)}$ is also UMD for all $\beta>\alpha$. This can easily be seen from the formula $(H f)^{2}=f^{2}+2 H(f H(f))$ (that allows us to pass from $\alpha$ to $2 \alpha$ ) and interpolation.

Now we turn to describe elementary notions from the interpolation theory (see [BL] for more information). Let $\left(X_{0}, X_{1}\right)$ be an interpolation couple of quasi-Banach spaces. We denote by $X_{0}+X_{1}$ and $X_{0} \cap X_{1}$ respectively the sum and intersection of $X_{0}$ and $X_{1}$. Let $t>0$ and $x \in X_{0}+X_{1}$. Define

$$
K\left(t, x ; X_{0}, X_{1}\right)=\inf \left\{\left\|x_{0}\right\|_{X_{0}}+t\left\|x_{1}\right\|_{X_{1}}: x=x_{0}+x_{1}, x_{j} \in X_{j}, j=0,1\right\} \text {. }
$$

This is the so-called $K$-functional. Given $0<\theta<1,0<q \leq \infty$, we define

$$
\|x\|_{\theta q}=\left(\int_{0}^{\infty}\left(t^{-\theta} K\left(t, x ; X_{0}, X_{1}\right)\right)^{q} \frac{d t}{t}\right)^{1 / q}, \quad x \in X_{0}+X_{1},
$$

and

$$
\left(X_{0}, X_{1}\right)_{\theta q}=\left\{x \in X_{0}+X_{1}:\|x\|_{\theta q}<\infty\right\} .
$$

Then $\left(X_{0}, X_{1}\right)_{\theta q}$, equipped with the quasi-norm $\|\cdot\|_{\theta q}$, is a quasi-Banach space. This is the real interpolation space of $X_{0}$ and $X_{1}$ with parameters $\theta$ and $q$.

There exists another (equivalent) way to construct this space by means of the so-called $J$-functional. Let for $t>0$

$$
J\left(t, x ; X_{0}, X_{1}\right)=\max \left(\|x\|_{X_{0}}, t\|x\|_{X_{1}}\right), \quad x \in X_{0} \cap X_{1} .
$$

Define

$$
\|x\|_{\theta q ; J}=\inf \left(\sum_{n=-\infty}^{\infty}\left(2^{-n \theta} J\left(2^{n}, x_{n} ; X_{0}, X_{1}\right)\right)^{q}\right)^{1 / q},
$$

where the infimum is taken over all representations of $x$ as

$$
x=\sum_{n=-\infty}^{\infty} x_{n}, \quad x_{n} \in X_{0} \cap X_{1}(n \in \mathbf{Z}),
$$


where the series converges in $X_{0}+X_{1}$. Then it is well known that

$$
C^{-1}\|x\|_{\theta q} \leq\|x\|_{\theta q ; J} \leq C\|x\|_{\theta q}, \quad \forall x \in X_{0}+X_{1},
$$

where $C$ is a constant depending only on $\theta$ on $q$. Therefore

$$
\left(X_{0}, X_{1}\right)_{\theta q}=\left\{x \in X_{0}+X_{1}:\|x\|_{\theta q ; J}<\infty\right\} \text {. }
$$

We shall also need interpolation spaces constructed by the complex method. The classical construction of Calderón [C] for Banach spaces needs some minor modifications for the quasi-Banach space setting (cf. e.g., [CMS]). Let $\mathscr{S}=$ $\{\zeta \in \mathbf{C}: 0<\operatorname{Re} \zeta<1\}$ and $\mathscr{A}$ be the family of the complex functions analytic in the strip $\mathscr{S}$, continuous and bounded on the closed strip $\overline{\mathscr{S}}$. Given an interpolation couple $\left(X_{0}, X_{1}\right)$ of quasi-Banach spaces, let

$$
\mathscr{F}\left(X_{0}, X_{1}\right)=\left\{\sum_{k=1}^{n} f_{k} x_{k}: f_{k} \in \mathscr{A}, x_{k} \in X_{0} \cap X_{1}, 1 \leq k \leq n, n \in \mathbf{N}\right\},
$$

and for $F \in \mathscr{F}\left(X_{0}, X_{1}\right)$

$$
\|F\|_{\mathscr{F}\left(X_{0}, X_{1}\right)}=\max \left\{\sup _{\eta \in \mathbf{R}}\|F(i \eta)\|_{X_{0}}, \sup _{\eta \in \mathbf{R}}\|F(1+i \eta)\|_{X_{1}}\right\} .
$$

Let $0<\theta<1$. We define

$$
\|x\|_{\theta}=\inf \left\{\|F\|_{\mathscr{F}_{\left(X_{0}, X_{1}\right)}}: F(\theta)=x, F \in \mathscr{F}\left(X_{0}, X_{1}\right)\right\}, \quad x \in X_{0} \cap X_{1} .
$$

Then $\|\cdot\|_{\theta}$ is a quasi-norm on $X_{0} \cap X_{1}$. The completion of $X_{0} \cap X_{1}$ with respect to it is denoted by $\left(X_{0}, X_{1}\right)_{\theta}$, which is the complex interpolation space of $X_{0}$ and $X_{1}$ with parameter $\theta$. It is well known that this definition coincides with the classical one if $X_{0}, X_{1}$ are Banach spaces (cf. [S]).

We shall freely use the following well-known results (cf. [BL]; these results are stated and proved in [BL] for Banach spaces. The proofs there, however, easily extend to quasi-Banach space setting). Let $\left(X_{0}, X_{1}\right)$ be an interpolation couple of quasi-Banach spaces and $0<\theta<1,0<p_{0}, p_{1} \leq \infty$. Then

$$
\begin{aligned}
& \left(\widetilde{L}^{p_{0}}\left(X_{0}\right), \widetilde{L}^{p_{1}}\left(X_{1}\right)\right)_{\theta p}=\widetilde{L}^{p}\left(\left(X_{0}, X_{1}\right)_{\theta p}\right), \\
& \left(\widetilde{L}^{p_{0}}\left(X_{0}\right), \widetilde{L}^{p_{1}}\left(X_{1}\right)\right)_{\theta}=\widetilde{L}^{p}\left(\left(X_{0}, X_{1}\right)_{\theta}\right),
\end{aligned}
$$

where $\frac{1}{p}=\frac{1-\theta}{p_{0}}+\frac{\theta}{p_{1}}$. If $X_{0}$ and $X_{1}$ are quasi-Banach lattices of measurable functions, we can easily show that the above equalities hold also for the couple $\left(L^{p_{0}}\left(X_{0}\right), L^{p_{1}}\left(X_{1}\right)\right)$. We shall see that for many couples of quasi-Banach lattices, Hardy space versions of these results hold. We have chosen to work with the interpolation couple $\left(H^{p_{0}}\left(E_{0}\right), H^{p_{1}}\left(E_{1}\right)\right)$ rather than $\left(\widetilde{H}^{p_{0}}\left(E_{0}\right), \widetilde{H}^{p_{1}}\left(E_{1}\right)\right)$ for the sake of simplicity. Since we proceed with the help of certain explicit formulae, all the results stated below transfer to the couple $\left(\widetilde{H}^{p_{0}}\left(E_{0}\right), \widetilde{H}^{p_{1}}\left(E_{1}\right)\right)$ with essentially the same proofs; the case of the spaces with tilde would, however, require routine but somewhat nasty discussions of approximability by simple functions that we prefer to avoid.

\section{NECESSITY OF THE BMO-CONDITION}

The main result of this section is the following theorem. We shall use the convention that $L^{\infty}(w)=L^{\infty}, H^{\infty}(w)=H^{\infty}, w^{1 / \infty}=1$. 
Theorem 2.1. Let $w_{0}, w_{1}$ be weights on $\mathbf{T}$ such that $\log w_{j} \in L^{1} \quad(j=0,1)$ and let $0<p_{0}, p_{1} \leq \infty$. If there exists $0<\theta<1$ such that

$$
H^{p}(w) \subset\left(H^{p_{0}}\left(w_{0}\right), H^{p_{1}}\left(w_{1}\right)\right)_{\theta \infty}, \quad \text { (continuous inclusion), }
$$

where $\frac{1}{p}=\frac{1-\theta}{p_{0}}+\frac{\theta}{p_{1}}$ and $w=w_{0}^{p(1-\theta) / p_{0}} w_{1}^{p \theta / p_{1}}$, then $\log \left(w_{0}^{1 / p_{0}} w_{1}^{-1 / p_{1}}\right) \in \mathrm{BMO}$.

The reader is referred to $[\mathrm{G}, \mathrm{GR}]$ or $[\mathrm{T}]$ for the definition of BMO.

Since $\left(X_{0}, X_{1}\right)_{\theta p} \subset\left(X_{0}, X_{1}\right)_{\theta \infty}$ for an arbitrary couple of quasi-Banach spaces $\left(X_{0}, X_{1}\right)$, Theorem 2.1 implies immediately that the BMO-condition is necessary for the first equality in (0.2). It is also necessary for the second equality if $p_{0}, p_{1} \geq 1$ (because $\left(X_{0}, X_{1}\right)_{\theta} \subset\left(X_{0}, X_{1}\right)_{\theta \infty}$ if $X_{0}$ and $X_{1}$ are Banach spaces). We conjecture that this last restriction on $p_{0}, p_{1}$ is in fact irrelevant.

Proof of Theorem 2.1. We shall exploit an idea from [CMW]. Suppose that one of $p_{0}$ and $p_{1}$ is not infinite (otherwise there is nothing to prove), say $p_{0}<\infty$. Multiplying all the spaces by an outer function with modulus $w_{1}^{1 / p_{1}}$, we can, and do assume $w_{1} \equiv 1$.

For $t>0$, let $\varphi_{t}$ and $\psi_{t}$ be outer functions such that $\left|\varphi_{t}\right|=\min \left(1, t w_{0}^{1 / p_{0}}\right)$, $\left|\psi_{t}\right|=\min \left(1, t^{-1} w_{0}^{-1 / p_{0}}\right)$, a.e. on $\mathbf{T}$. Now suppose $\log w_{0}^{1 / p_{0}} \notin \mathrm{BMO}$. Then by Lemma 1.2 in [CMW], for every $\varepsilon>0$ there exist $t>0$ and $z \in D$ such that

$$
\left|\varphi_{t}(z)\right|+\left|\psi_{t}(z)\right|<\varepsilon
$$

(avoiding to reproduce the proof of that, we mention only that it is short and is based on exploiting the Garcia norm on BMO). Considering $t^{p_{0}} w_{0}$ instead of $w_{0}$, we may assume $t=1$. Let $f \in H^{p}(w)$ with $\|f\|_{H^{p}(w)}<1$. By (2.1), for any $s>0$ there exist $f_{0} \in H^{p_{0}}\left(w_{0}\right)$ and $f_{1} \in H^{p_{1}}$ such that $f=f_{0}+f_{1}$ and

$$
\left\|f_{0}\right\|_{H^{p_{0}}\left(w_{0}\right)} \leq C_{0} s^{\theta} \text { and }\left\|f_{1}\right\|_{H^{p_{1}}} \leq C_{0} s^{\theta-1}
$$

where $C_{0}$ is the norm of the inclusion (2.1). Let

$$
F=\left(\varphi_{1} \psi_{1}^{-1}\right)^{1-\theta} f, \quad F_{0}=\varphi_{1} \psi_{1}^{-1} f_{0}, \quad F_{1}=f_{1} .
$$

Then $F \in H^{p}, F_{j} \in H^{p_{j}}(j=0,1)$ and

$$
\begin{gathered}
\|F\|_{H^{p}}<1, \quad\left\|F_{0}\right\|_{H^{p_{0}}} \leq C_{0} s^{\theta}, \quad\left\|F_{1}\right\|_{H^{p_{1}}} \leq C_{0} s^{\theta-1} \\
\left(\psi_{1} \varphi_{1}^{-1}\right)^{1-\theta} F=\left(\psi_{1} \varphi_{1}^{-1}\right) F_{0}+F_{1} .
\end{gathered}
$$

Let $P_{z}$ denote the Poisson kernel of the unit disc corresponding to $z$. Then we obtain

$$
\begin{aligned}
\left|F_{0}(z)\right| & \leq \exp \int_{\mathbf{T}} \log \left|F_{0}\right| P_{z} d m \\
& \leq \exp \left\{\int_{\left|\varphi_{1}\right|<1} \log \left(\left|\varphi_{1}\right|^{\theta}|F|+\left|\varphi_{1}\right|\left|F_{1}\right|\right) P_{z} d m+\int_{\left|\varphi_{1}\right|=1} \log \left|F_{0}\right| P_{z} d m\right\} \\
& \leq\left|\varphi_{1}(z)\right|^{\theta} \exp \int_{\mathbf{T}} \log \left(|F|+\left|F_{0}\right|+\left|F_{1}\right|\right) P_{z} d m .
\end{aligned}
$$


Now let $r=\min \left(p_{0}, p_{1}\right)$. Then by Jensen and Hölder inequalities and (2.3)

$$
\begin{aligned}
& \exp \int_{\mathbf{T}} \log \left(|F|+\left|F_{0}\right|+\left|F_{1}\right|\right) P_{z} d m \\
& \quad \leq\left(\int_{\mathbf{T}}\left(|F|+\left|F_{0}\right|+\left|F_{1}\right|\right)^{r} P_{z} d m\right)^{1 / r} \\
& \quad \leq C\left[(1-|z|)^{-1 / p}+s^{\theta}(1-|z|)^{-1 / p_{0}}+s^{\theta-1}(1-|z|)^{-1 / p_{1}}\right],
\end{aligned}
$$

where $C$ is a constant depending on $C_{0}, p$ and $p_{j}(j=0,1)$. Therefore

$$
\left|F_{0}(z)\right| \leq C\left|\varphi_{1}(z)\right|^{\theta}\left[(1-|z|)^{-1 / p}+s^{\theta}(1-|z|)^{-1 / p_{0}}+s^{\theta-1}(1-|z|)^{-1 / p_{1}}\right] .
$$

Similarly

$$
\left|F_{1}(z)\right| \leq C\left|\psi_{1}(z)\right|^{1-\theta}\left[(1-|z|)^{-1 / p}+s^{\theta}(1-|z|)^{-1 / p_{0}}+s^{\theta-1}(1-|z|)^{-1 / p_{1}}\right] .
$$

Combining the preceding inequalities with (2.4) we obtain

$$
\begin{aligned}
|F(z)| \leq\left|\psi_{1}(z) \varphi_{1}(z)^{-1}\right|^{\theta}\left|F_{0}(z)\right|+\left|\psi_{1}(z) \varphi_{1}(z)^{-1}\right|^{\theta-1}\left|F_{1}(z)\right| & \\
\leq C\left(\left|\psi_{1}(z)\right|^{\theta}+\left|\varphi_{1}(z)\right|^{1-\theta}\right)\left[(1-|z|)^{-1 / p}\right. & \left.\quad+s^{\theta}(1-|z|)^{-1 / p_{0}}+s^{\theta-1}(1-|z|)^{-1 / p_{1}}\right] .
\end{aligned}
$$

Now note that $F$ can be an arbitrary element of the unit ball of $H^{p}$, so $\sup _{F}|F(z)| \geq\left(1-|z|^{2}\right)^{-1 / p}$ (see the remark below). Taking into account (2.2) and setting $s=(1-|z|)^{1 / p_{0}}(1-|z|)^{-1 / p_{1}}$, we arrive at

$$
\left(1-|z|^{2}\right)^{-1 / p} \leq 3 C\left(\varepsilon^{\theta}+\varepsilon^{1-\theta}\right)(1-|z|)^{-1 / p},
$$

which yields a contradiction if $\varepsilon$ is sufficiently small. This proves Theorem 2.1 .

Remark. Let $z \in D$ and $\Phi_{p, z}$ be the evaluation functional at $z$ defined on $H^{p}$ by $\Phi_{p, z}(F)=F(z)$. In the above proof, we implicitly used the elementary fact that the norm of $\Phi_{p, z}$ is of the same order as $(1-|z|)^{-1 / p}$. In fact, we precisely have that $\left\|\Phi_{p, z}\right\|=\left(1-|z|^{2}\right)^{-1 / p}$. This is easy to prove. Indeed, by factorization, it suffices to show this for $p=2$. Then consider the Cauchy kernel $\Psi(\zeta)=\frac{1}{1-\bar{z} \zeta}$. We have

$$
\int_{\mathbf{T}} F(\zeta) \overline{\Psi(\zeta)} d m(\zeta)=F(z), \quad \forall F \in H^{2}
$$

Thus it follows that $\left\|\Phi_{2, z}\right\|=\|\Psi\|_{H^{2}}=\left(1-|z|^{2}\right)^{-1 / 2}$.

We give an immediate consequence of Theorem 2.1. It is of interest in connection with the analytic projection constructed in [B2] (see also [K2, K4] for another construction).

Corollary 2.2. Let $w$ be a weight such that $\log w \in L^{1}$. Suppose that there exists an operator $Q$ projecting boundedly $L^{p_{0}}(w)$ onto $H^{p_{0}}(w)$ and at the same time $L^{p_{1}}(w)$ onto $H^{p_{1}}(w)$ for some $1<p_{0}, p_{1}<\infty, p_{0} \neq p_{1}$. Then $\log w \in$ BMO.

Note that, conversely, if $\log w \in \mathrm{BMO}$, then one can easily construct a projection $Q$ from $L^{p}(w)$ onto $H^{p}(w)$ that is continuous for $p$ in a certain interval $(a, b)$. One can vary $a$ and $b$ (not independently). In particular, it 
is possible to take $a=1$. It is not clear at the time of this writing if one can always ensure $(a, b)=(1, \infty)$.

\section{Weighted HARDY SPACES: THE REAL INTERPOLATION}

The main result of this section is the following theorem.

Theorem 3.1. Let $w_{0}$ and $w_{1}$ be weights on $\mathbf{T}$ such that $\log w_{j} \in L^{1} \quad(j=$ $0,1)$. Let $0<p_{0}, p_{1} \leq \infty$. If $\log \left(w_{0}^{1 / p_{0}} w_{1}^{-1 / p_{1}}\right) \in \mathrm{BMO}$, then there exists $a$ constant $C$ depending only on $w_{j}$ and $p_{j}(j=0,1)$ such that for any $t>0$ and any $f \in H^{p_{0}}\left(w_{0}\right)+H^{p_{1}}\left(w_{1}\right)$

$$
K\left(t, f ; H^{p_{0}}\left(w_{0}\right), H^{p_{1}}\left(w_{1}\right)\right) \leq C K\left(t, f ; L^{p_{0}}\left(w_{0}\right), L^{p_{1}}\left(w_{1}\right)\right) .
$$

Remark. Note that in the case $p_{0}=p_{1}<\infty$ Theorem 3.1 has already been proved in [CMW]. The proof of [CMW] does not seem to be adaptable to give Theorem 3.1 for $p_{0} \neq p_{1}$. The same remark applies to Theorem 4.1 below on the complex interpolation of weighted Hardy spaces.

Before proving Theorem 3.1, we give an immediate consequence of it. Note first that the reverse inequality to $(3.1)$ is evident (with $C=1$ ). Recall also that for $0<\theta<1$

$$
\left(L^{p_{0}}\left(w_{0}\right), L^{p_{1}}\left(w_{1}\right)\right)_{\theta p}=L^{p}(w),
$$

where $\frac{1}{p}=\frac{1-\theta}{p_{0}}+\frac{\theta}{p_{1}}$ and $w=w_{0}^{p(1-\theta) / p_{0}} w_{1}^{p \theta / p_{1}}$ (cf. [BL]).

Corollary 3.2. Under the same assumptions as in Theorem 3.1, for any given $0<$ $\theta<1$ and $0<q \leq \infty$, the quasi-norm on $\left(H^{p_{0}}\left(w_{0}\right), H^{p_{1}}\left(w_{1}\right)\right)_{\theta q}$ is equivalent to that induced on it by the quasi-norm of $\left(L^{p_{0}}\left(w_{0}\right), L^{p_{1}}\left(w_{1}\right)\right)_{\theta q}$. Consequently

$$
\left(H^{p_{0}}\left(w_{0}\right), H^{p_{1}}\left(w_{1}\right)\right)_{\theta p}=H^{p}(w),
$$

where $\frac{1}{p}=\frac{1-\theta}{p_{0}}+\frac{\theta}{p_{1}}$ and $w=w_{0}^{p(1-\theta) / p_{0}} w_{1}^{p \theta / p_{1}}$.

Proof of Theorem 3.1. To exclude the trivial case $p_{0}=p_{1}=\infty$, we assume that $0<p_{0}<\infty$ and $0<p_{1} \leq \infty$. After multiplying all the spaces by an outer function with modulus $w_{1}^{1 / p_{1}}$, we may, and do assume $w_{1} \equiv 1$ and $\log w_{0} \in$ BMO. It is well known that $\log w_{0} \in$ BMO iff there exist $\gamma>0$ and $v \in A_{2}$ such that $w_{0}=v^{\gamma}$, both $\gamma$ and the $A_{2}$-constant for $v$ depending on the BMO-norm of $\log w_{0}$ only (cf. [GR, T]). We factorize $v$ as $v=v_{0} v_{1}^{-1}$ with $v_{0}, v_{1} \in A_{1}$, and then apply Lemma 1.1 to $K=k p_{0} / \gamma$, with $k$ a sufficiently large integer (we need, among other things, $k p_{0} \geq 2 \gamma$, since $K \geq 2$ ). We obtain a representation $w_{0}=\left(u_{0} u_{1}^{-1}\right)^{2 k p_{0}}$ with

(3.2) $\left|H\left(u_{0}\right)\right| \leq C u_{0}$

(3.3) $M\left(u_{j}^{2}\right) \leq C u_{j}^{2}(j=0,1)$.

We need the following majoration lemma.

Lemma 3.3. If $k$ is so large that $k p_{1} \geq 1$, then for every positive $a \neq 0$ in $L^{p_{1}}$ there exists a positive function $b$ bounded away from zero and satisfying

(3.4) $b \geq a$, a.e. on $\mathbf{T}$;

(3.5) $\|b\|_{L^{p_{1}}} \leq C\|a\|_{L^{p_{1}}}$

(3.6) $\left|H\left(u_{0} b^{1 / 2 k}\right)\right| \leq C u_{0} b^{1 / 2 k}$, a.e. on $\mathrm{T}$.

Proof. If $p_{1}=\infty$, we take simply $b \equiv\|a\|_{\infty}$, then (3.6) follows from (3.2). Otherwise we set $a_{0}=(a+\varepsilon)^{1 / 2 k}$ with $\varepsilon>0$ very small and, inductively, 
$a_{n}=u_{0}^{-1}\left|H\left(u_{0} a_{n-1}\right)\right|, n \geq 1$. By (3.3) and "if" part of Jones' factorization theorem, $u_{0}^{-2 k p_{1}}=\left(u_{0}^{2}\right)^{1-\left(1+k p_{1}\right)} \in A_{1+k p_{1}}$. Since $2 k p_{1} \geq 1+k p_{1}>1, H$ is bounded on $L^{2 k p_{1}}\left(u_{0}^{-2 k p_{1}}\right)$; so there exists a constant $C$ (independent of $a$ ) such that

$$
\left\|a_{n}\right\|_{L^{2 k p_{1}}} \leq C\left\|a_{n_{1}}\right\|_{L^{2 k p_{1}}} \leq \cdots \leq C^{n}\left\|a_{0}\right\|_{L^{2 k p_{1}}} \leq C^{n}\|a\|_{L^{p_{1}}}^{1 / 2 k} .
$$

It is straightforward that the function $b$ defined by

$$
b=\left(\sum_{n \geq 0}(2 C)^{-n} a_{n}\right)^{2 k}
$$

enjoys the desired properties.

The following lemma is the crucial point of the present proof.

Lemma 3.4. Let $f_{0} \in L^{p_{0}}\left(w_{0}\right), f_{1} \in L^{p_{1}}$. Then there exists a function $G \in H^{\infty}$ such that

$$
\begin{aligned}
& \max \left\{\left\|(1-G) f_{0}\right\|_{L^{p_{0}}\left(w_{0}\right)},\left\|(1-G) f_{1}\right\|_{L^{p_{0}}\left(w_{0}\right)}\right\} \leq C\left\|f_{0}\right\|_{L^{p_{0}}\left(w_{0}\right)}, \\
& \max \left\{\left\|G f_{0}\right\|_{L^{p_{1}}},\left\|G f_{1}\right\|_{L^{p_{1}}}\right\} \leq C\left\|f_{1}\right\|_{L^{p_{1}}},
\end{aligned}
$$

where $C$ is a constant depending only on $w_{0}, p_{0}$ and $p_{1}$.

Supposing that this lemma has already been verified, we can finish the proof of Theorem 3.1 as follows. Let $t>0$ and $f \in H^{p_{0}}\left(w_{0}\right)+H^{p_{1}}$. Take $f_{0} \in$ $L^{p_{0}}\left(w_{0}\right)$ and $f_{1} \in L^{p_{1}}$ such that $f=f_{0}+f_{1}$ and

$$
\left\|f_{0}\right\|_{L^{p_{0}}\left(w_{0}\right)}+t\left\|f_{1}\right\|_{L^{p_{1}}} \leq 2 K\left(t, f ; L^{p_{0}}\left(w_{0}\right), L^{p_{1}}\right) .
$$

Applying Lemma 3.4 to $f_{0}$ and $f_{1}$, we find $G \in H^{\infty}$ satisfying (3.7) and (3.8). Then $f=(1-G) f+G f=g+h$, where $g=(1-G) f, h=G f$. By (3.7)

$$
\begin{aligned}
\|g\|_{L^{p_{0}}\left(w_{0}\right)} & \leq C\left(\left\|(1-G) f_{0}\right\|_{L^{p_{0}}\left(w_{0}\right)}+\left\|(1-G) f_{1}\right\|_{L^{p_{0}}\left(w_{0}\right)}\right) \\
& \leq C\left\|f_{0}\right\|_{L^{p_{0}}\left(w_{0}\right)} .
\end{aligned}
$$

Similarly, by (3.8) $\|h\|_{L^{p_{1}}} \leq C\left\|f_{1}\right\|_{L^{p_{1}}}$. Since $f, G$ are analytic, we deduce that $g \in H^{p_{0}}\left(w_{0}\right), h \in H^{p_{1}}$ and

$$
\begin{aligned}
\|g\|_{H^{p_{0}}\left(w_{0}\right)}+t\|h\|_{H^{p_{1}}} & \leq C\left(\left\|f_{0}\right\|_{L^{p_{0}}\left(w_{0}\right)}+t\left\|f_{1}\right\|_{L^{p_{1}}}\right) \\
& \leq 2 C K\left(t, f ; L^{p_{0}}\left(w_{0}\right), L^{p_{1}}\right) .
\end{aligned}
$$

This shows (3.1).

It remains to prove Lemma 3.4. Let $k$ be a large integer such that $k p_{0} \geq 1$ and $k p_{1} \geq 1$ (and also $k p_{0} \geq 2 \gamma$, that has been supposed from the very beginning). Let $b$ be the function given by Lemma 3.3 when applied to $a=$ $\left|f_{1}\right|$. Define

$$
\begin{aligned}
\alpha & =\max \left\{1,\left|f_{0} b^{-1}\right|^{1 / 2 k}\right\}, \\
F_{1} & =u_{0} b^{1 / 2 k}+i H\left(u_{0} b^{1 / 2 k}\right), \\
F_{2} & =u_{0} b^{1 / 2 k} \alpha+i H\left(u_{0} b^{1 / 2 k} \alpha\right) .
\end{aligned}
$$

By Remark 1.2, $u_{0} b^{1 / 2 k}, u_{0} b^{1 / 2 k} \alpha \in L^{2}$ if $k$ is large enough; $k$ will then be assumed to ensure that property. Then, $F_{1}, F_{2}$ are in $H^{2}$ and their real parts 
are strictly positive a.e. on $\mathrm{T}$. Hence the function $F=F_{1} F_{2}^{-1}$ is analytic. By (3.6) $\left|F_{1}\right| \leq(1+C) u_{0} b^{1 / 2 k}$. On the other hand, $\left|F_{2}\right| \geq u_{0} b^{1 / 2 k} \alpha$. Therefore, $|F| \leq C \alpha^{-1}$. It follows that $F \in H^{\infty}$ and $\|F\|_{H^{\infty}} \leq C$. Now define $G=$ $1-\left(1-F^{2 k}\right)^{2 k}$. Then $G \in H^{\infty}$ and $|G| \leq C|F|^{2 k} \leq C \alpha^{-2 k}$. In particular, $\|G\|_{H^{\infty}} \leq C$, and thus to check (3.7) and (3.8) it suffices to show that

$$
\left\|G f_{0}\right\|_{L^{p_{1}}} \leq C\left\|f_{1}\right\|_{L^{p_{1}}}, \quad\left\|(1-G) f_{1}\right\|_{L^{p_{0}}\left(w_{0}\right)} \leq C\left\|f_{0}\right\|_{L^{p_{0}}\left(w_{0}\right)} \text {. }
$$

The first inequality is easy to prove. Indeed

$$
\left|G f_{0}\right| \leq C\left|f_{0}\right| \alpha^{-2 k} \leq C b
$$

and it is sufficient to refer to (3.5). For the second inequality, note that $\left|(1-G) f_{1}\right| \leq C|1-F|^{2 k} b$. Now

$$
\begin{aligned}
|1-F|^{2 k} b & =\left|\left[u_{0} b^{1 / 2 k}(\alpha-1)+i H\left(u_{0} b^{1 / 2 k}(\alpha-1)\right)\right] F_{2}^{-1}\right|^{2 k} b \\
& \leq C\left[|\alpha-1|^{2 k} b+\left|H\left(u_{0} b^{1 / 2 k}(\alpha-1)\right)\right|^{2 k} u_{0}^{-2 k}\right] .
\end{aligned}
$$

Therefore

$$
\begin{aligned}
& \left\|(1-G) f_{1}\right\|_{L^{p_{0}}\left(w_{0}\right)} \\
& \quad \leq C\left[\left\|(\alpha-1)^{2 k} b\right\|_{L^{p_{0}}\left(w_{0}\right)}+\left\|\left|H\left(u_{0} b^{1 / 2 k}(\alpha-1)\right)\right|^{2 k} u_{0}^{-2 k}\right\|_{L^{p_{0}}\left(w_{0}\right)}\right] .
\end{aligned}
$$

Since $w_{0}=\left(u_{0} u_{1}^{-1}\right)^{2 k p_{0}}$, we see that the second term in the brackets on the right is

$$
\left\|H\left(u_{0} b^{1 / 2 k}(\alpha-1)\right)\right\|_{L^{2 k p_{0}\left(u_{1}^{-2 k p_{0}}\right)}}^{2 k} \text {. }
$$

By (3.3), $u_{1}^{-2 k p_{0}} \in A_{1+k p_{0}}$ and thus $H$ is bounded on $L^{2 k p_{0}}\left(u_{1}^{-2 k p_{0}}\right)$. It follows that the second term on the right in (3.9) is majorized by the first one. Since $(\alpha-1)^{2 k} b \leq\left|f_{0}\right|$, we obtain the desired estimate

which concludes the proof.

$$
\left\|(1-G) f_{1}\right\|_{L^{p_{0}\left(w_{0}\right)}} \leq C\left\|f_{0}\right\|_{L^{p_{0}}\left(w_{0}\right)},
$$

Remarks. (i) The above proof of Theorem 3.1 shows in fact that the constant $C$ in (3.1) depends only on $p_{0}, p_{1}$ and the norm of $\log \left(w_{0}^{1 / p_{0}} w_{1}^{-1 / p_{1}}\right)$ in BMO.

(ii) Lemma 3.4 gives also a similar $J$-functional estimate for the couple of the quotient spaces

$$
\left(\frac{L^{p_{0}}\left(w_{0}\right)}{H^{p_{0}}\left(w_{0}\right)}, \frac{L^{p_{1}}\left(w_{1}\right)}{H^{p_{1}}\left(w_{1}\right)}\right) .
$$

Explicitly, we have a constant $C$ such that for any $t>0$ and any $\tilde{f}$ in the intersection of these quotient spaces there exists $f \in L^{p_{0}}\left(w_{0}\right) \cap L^{p_{1}}\left(w_{1}\right)$ which represents the class $\tilde{f}$ simultaneously in both quotient spaces and satisfies

$$
J\left(t, f ; L^{p_{0}}\left(w_{0}\right), L^{p_{1}}\left(w_{1}\right)\right) \leq C J\left(t, \tilde{f} ; \frac{L^{p_{0}}\left(w_{0}\right)}{H^{p_{0}}\left(w_{0}\right)}, \frac{L^{p_{1}}\left(w_{1}\right)}{H^{p_{1}}\left(w_{1}\right)}\right) .
$$

Note, however, that this latter $J$-functional estimate for the quotient spaces is in fact equivalent to the $K$-functional estimate (3.1) described in Theorem 3.1. This follows from a simple but very useful observation of Pisier [P2] stating that such $K$ - and $J$-functional estimates are formally equivalent in the general situation of two couples $\left(A_{0}, A_{1}\right) \subset\left(X_{0}, X_{1}\right)$.

\section{Weighted HARDY SPACES: THE COMPLEX INTERPOLATION}

In this section, we shall prove the following counterpart of Corollary 3.2 for the complex interpolation. 
Theorem 4.1. Let $0<p_{0}, p_{1} \leq \infty$ and $w_{0}, w_{1}$ be weights such that $\log w_{j} \in L^{1}$ $(j=0,1)$. If $\log \left(w_{0}^{1 / p_{0}} w_{1}^{-1 / p_{1}}\right) \in \mathrm{BMO}$, then for every $0<\theta<1$

$$
\left(H^{p_{0}}\left(w_{0}\right), H^{p_{1}}\left(w_{1}\right)\right)_{\theta}=H^{p}(w),
$$

where $\frac{1}{p}=\frac{1-\theta}{p_{0}}+\frac{\theta}{p_{1}}$ and $w=w_{0}^{p(1-\theta) / p_{0}} w_{1}^{p \theta / p_{1}}$.

To prove Theorem 4.1, we need the following technical lemma on the existence of certain analytic decompositions of unity, which is of independent interest.

Lemma 4.2. Let $0<p \leq \infty$ and $w$ be a weight such that $\log w \in \mathrm{BMO}$. Then for every positive function $a \in L^{p}(w)$ there exist $b \in L^{p}(w)$ and a sequence $\left\{\varphi_{n}\right\}_{n \in \mathrm{Z}} \subset H^{\infty}$ satisfying the following properties

(4.2) $b \geq a$, a.e. on $\mathrm{T}$;

(4.3) $\|b\|_{L^{p}(w)} \leq C\|a\|_{L^{p}(w)}$;

(4.4) $\left\|\varphi_{n}\right\|_{H^{\infty}} \leq C, \forall n \in \mathbf{Z}$;

(4.5) $\left|\varphi_{n}\right|^{1 / 4} b \leq C 2^{n}$, a.e. on $\mathbf{T}, \forall n \in \mathbf{Z}$;

(4.6) $\sum_{n \in Z}\left|\varphi_{n}\right|^{1 / 4} 2^{n} \leq C b$, a.e. on $\mathrm{T}$;

(4.7) $\sum_{n \in \mathbf{Z}} \varphi_{n}=1$, a.e. on $\mathbf{T}$,

where $C$ is a constant depending only on $w$ on $p$.

Before passing to the proof of the lemma we note that (4.5)-(4.7) mean that the $\varphi_{n}$ 's behave roughly as the functions $\chi_{\left\{2^{n-1}<b \leq 2^{n}\right\}}$, but have an advantage of being analytic. Here and in the sequel, $\chi_{e}$ denotes the characteristic function of a subset $e \subset \mathbf{T}$.

Proof. Take a large integer $k$ (we need, among other things, $k p \geq 1$ ) and represent, as in $\S 3$, the weight $w$ in the form $w=\left(u_{0} u_{1}^{-1}\right)^{2 k p}$ so that (3.2) and (3.3) hold.

We assume first that $a$ is bounded, say $a<2^{N}$ a.e. on $\mathbf{T}$ for some integer $N$. We now define by induction two sequences $\left\{G_{n}\right\}_{n \leq N} \subset H^{\infty}$ and $\left\{b_{n}\right\}_{n \leq N} \subset$ $L^{\infty}$ as follows.

Let $G_{N} \equiv 1, b_{N}=a$. Then define inductively for $n \leq N-1$

$$
\begin{aligned}
\alpha_{n} & =\max \left\{1,\left(b_{n+1} 2^{-n}\right)^{1 / 4 k}\right\}, \\
F_{n} & =\frac{u_{0}+i H u_{0}}{\alpha_{n} u_{0}+i H\left(\alpha_{n} u_{0}\right)}, \quad G_{n}=1-\left(1-F_{n}^{16 k}\right)^{8 k}, \\
b_{n} & =b_{n+1}+\delta\left|G_{n+1}-G_{n}\right|^{1 / 4} 2^{n+1},
\end{aligned}
$$

where $\delta \in(0,1)$ is a constant to be specified later ( $\delta$ will not depend on $a, N)$.

By using (3.2) and Remark 1.2, we easily check, as in the proof of Lemma 3.4 , that $F_{n}, G_{n} \in H^{\infty}$ (if $k$ is large enough) and, moreover

$$
\left|F_{n}\right| \leq C \alpha_{n}^{-1} \leq C, \quad\left|G_{n}\right| \leq C\left|F_{n}\right|^{16 k} \leq C \alpha_{n}^{-16 k} \leq C .
$$

Define $\varphi_{n}=G_{n}-G_{n-1}(n \leq N)$. Then $\varphi_{n} \in H^{\infty}$ and $\left\|\varphi_{n}\right\|_{H^{\infty}} \leq C$.

From the definition of $b_{n}$

$$
a \leq b_{n+1} \leq b_{n} \leq b_{n+1}+C 2^{n}, \quad \forall n \leq N .
$$

It then follows that $\left\{b_{n}\right\}_{n \leq N}$ is a decreasing sequence of positive bounded functions on $\mathbf{T}$. Denote by $\bar{b}$ the a.e. limit of this sequence as $n \rightarrow-\infty$. Then 
clearly

$$
a \leq b \leq b_{n}+C 2^{n}, \quad \forall n \leq N
$$

which gives, in particular, (4.2).

We are going to check that the functions $b,\left\{\varphi_{n}\right\}_{n}$ just defined satisfy (4.2)(4.7). We have already verified (4.2) and (4.4). Now by (4.10), for $n \leq N$

$$
\begin{aligned}
\left|\varphi_{n}\right|^{1 / 4} b & \leq\left|G_{n}\right|^{1 / 4}\left(b_{n+1}+C 2^{n+1}\right)+\left|G_{n-1}\right|^{1 / 4}\left(b_{n}+C 2^{n}\right) \\
& \leq\left|G_{n}\right|^{1 / 4} b_{n+1}+\left|G_{n-1}\right|^{1 / 4} b_{n}+C 2^{n} \leq C 2^{n},
\end{aligned}
$$

in view of (4.8) and the definition of $\alpha_{n}$. This proves (4.5). It follows from (4.5) that the series $\sum_{n \leq N} \varphi_{n}$ converges absolutely a.e. on $\mathbf{T}$. Its sum is evidently equal to 1 a.e., that is, (4.7) holds. Hence, it remains to check (4.3) and (4.6).

Fix $n \leq N$. We are going to estimate $\left\|b_{n}\right\|_{L^{p}(w)}$. We assume for the moment $p<\infty$. We have

$$
b_{n}=a+\delta \sum_{m=n+1}^{N}\left|\varphi_{m}\right|^{1 / 4} 2^{m}
$$

and

$$
\begin{aligned}
& \sum_{m=n+1}^{N}\left|\varphi_{m}\right|^{1 / 4} 2^{m} \leq \sum_{m=n+1}^{N}\left(\left|1-G_{m}\right|^{1 / 4}+\left|1-G_{m-1}\right|^{1 / 4}\right) 2^{m} \leq C \sum_{m=n}^{N-1}\left|1-F_{m}\right|^{2 k} 2^{m} \\
& \leq C \sum_{m=n}^{N-1}\left|\alpha_{m}-1\right|^{2 k} 2^{m}+C \sum_{m=n}^{N-1}\left|H\left(u_{0}\left(\alpha_{M}-1\right)\right)\right|^{2 k} 2^{m} u_{0}^{-2 k}
\end{aligned}
$$

Now it is convenient to employ the weighted space $L^{2 k p}\left(l^{2 k} ; u_{1}^{-2 k p}\right)$ of $l^{2 k}$ valued functions. Clearly

$$
\begin{aligned}
& \left\|\sum_{m=n}^{N-1}\left|H\left(u_{0}\left(\alpha_{m}-1\right)\right)\right|^{2 k} 2^{m} u_{0}^{-2 k}\right\|_{L^{p}(w)} \\
& \quad=\left\|\left\{H\left(2^{m / 2 k} u_{0}\left(\alpha_{m}-1\right)\right)\right\}_{m=n}^{N-1}\right\|_{L^{2 k p}\left(l^{2 k} ; u_{1}^{-2 k p}\right)^{2 k}}
\end{aligned}
$$

From (3.3), $u_{1}^{2} \in A_{1}$; so $u_{1}^{-2 k p} \in A_{1+k p}$. By the choice of $k$, we have $2 k p \geq$ $1+k p$. Since also $2 k>1, H$ is bounded on $L^{2 k p}\left(l^{2 k} ; u_{1}^{-2 k p}\right)$ (cf. [AJ]). Thus, for some constant $C$

$$
\begin{aligned}
& \left\|\left\{H\left(2^{m / 2 k} u_{0}\left(\alpha_{m}-1\right)\right)\right\}_{m=n}^{N-1}\right\|_{L^{2 k p}\left(l^{2 k} ; u_{1}^{-2 k p}\right)} \\
& \quad \leq C\left\|\left\{2^{m / 2 k} u_{0}\left(\alpha_{m}-1\right)\right\}_{m=n}^{N-1}\right\|_{L^{2 k p}\left(l^{2 k} ; u_{1}^{-2 k p}\right)} \\
& \quad=C\left\|\sum_{m=n}^{N-1}\left(\alpha_{m}-1\right)^{2 k} 2^{m}\right\|_{L^{p}(w)}^{1 / 2 k} .
\end{aligned}
$$

Combining these estimates with (4.11), we obtain

$$
\left\|b_{n}\right\|_{L^{p}(w)} \leq C\|a\|_{L^{p}(w)}+C \delta\left\|\sum_{m=n}^{N-1}\left(\alpha_{n}-1\right)^{2 k} 2^{m}\right\|_{L^{p}(w)} .
$$


Now let $E_{N}=\varnothing, E_{n}=\left\{b_{n+1}>2^{n}\right\}$ for $n<N$ (note also that $E_{n}=\left\{\alpha_{n} \neq\right.$ $1\})$. Since $b_{n} \geq b_{n+1}$, it is clear that $E_{n} \supset E_{n+1}$. Denoting $e_{m}=E_{m} \backslash E_{m+1}$ $(m \leq N-1)$, it is easy to see that $2^{m} \leq b_{m} \leq C 2^{m}$ a.e. on $e_{m}$. It follows from (4.9) that

$$
2^{m} \leq b_{n} \leq C 2^{m} \text { a.e. on } e_{m}, \quad n \leq m \leq N-1,
$$

with $C$ independent of $m$ and $n$. Therefore

$$
\begin{aligned}
& \sum_{m=n}^{N-1}\left(\alpha_{m}-1\right)^{2 k} 2^{m}=\sum_{m=n}^{N-1}\left(\alpha_{m}-1\right)^{2 k} \chi_{E_{m}} 2^{m} \\
& \leq \sum_{m=n}^{N-1} 2^{m / 2} \sum_{j=m}^{N-1} b_{m+1}^{1 / 2} \chi_{e_{j}} \leq C \sum_{m=n}^{N-1} 2^{m / 2} \sum_{j=m}^{N-1} 2^{j / 2} \chi_{e_{j}} \\
& =C \sum_{j=n}^{N-1} 2^{j / 2} \chi_{e_{j}} \sum_{m=n}^{j} 2^{m / 2} \leq C^{\prime} \sum_{j=n}^{N-1} 2^{j} \chi_{e_{j}} \leq C^{\prime \prime} b_{n} .
\end{aligned}
$$

Hence by (4.12)

$$
\left\|b_{n}\right\|_{L^{p}(w)} \leq C\|a\|_{L^{p}(w)}+C \delta\left\|b_{n}\right\|_{L^{p}(w)} .
$$

Taking $\delta=(2 C)^{-1}$, we get finally

$$
\left\|b_{n}\right\|_{L^{p}(w)} \leq C\|a\|_{L^{p}(w)}, \quad \forall n \leq N-1,
$$

which then yields (4.3) by letting $n \rightarrow-\infty$ for $p<\infty$.

If $p=\infty,(4.3)$ is easy. Indeed, taking $l=\min \left\{n: a<2^{n}\right\}$, we clearly have $\alpha_{n} \equiv 1, F_{n} \equiv 1$ and $G_{n} \equiv 1$ for $n>l$. So $b$ will not exceed $C 2^{l}$ uniformly on $\mathbf{T}$.

From (4.5), we see that the series $\sum_{n \leq N}\left|\varphi_{n}\right|^{1 / 4} 2^{n}$ converges a.e. on $\mathbf{T}$; so letting $n \rightarrow-\infty$ in (4.11), we obtain

$$
b=a+\delta \sum_{n \leq N}\left|\varphi_{n}\right|^{1 / 4} 2^{n},
$$

which shows (4.6) with $C=\delta^{-1}$. This shows Lemma 4.2 in the case of bounded $a$.

The general case can be derived from this special one by a limit argument that we are going to describe now. Replacing $a$ by a slightly bigger function, we may assume that $a$ is bounded away from zero on $\mathbf{T}$. Then apply the result just proved to $a \wedge 2^{N}(N=1,2, \ldots)$. We obtain $\tilde{b}_{N},\left\{\varphi_{n N}\right\}_{n \leq N}$ satisfying (4.2)-(4.7) with $a \wedge 2^{N}$ instead of $a$. From (4.2) and (4.3) we deduce that $\log \tilde{b}_{N} \in L_{1}$. Let $B_{N}$ be an outer function with modulus $\tilde{b}_{N}$. Then (4.3) shows that $B_{N} \in H^{p}(w)$ and

$$
\left\|B_{N}\right\|_{H^{p}(w)} \leq C\left\|a \wedge 2^{N}\right\|_{L^{p}(w)} \leq C\|a\|_{L^{p}(w)} .
$$

Denote by $\varphi$ an outer function with modulus $w^{1 / p}$. We see that $\left\{\varphi B_{N}\right\}$ is a bounded sequence in $H^{p}$. So passing to a subsequence if necessary, we may assume that $\left\{\varphi B_{N}\right\}$ converges to some analytic function $G$ uniformly on every compact subset of $D$. Define $B=G \varphi^{-1}$. Then we deduce that $B_{N}$ converges to $B$ uniformly on every compact subset of $D$. Let $b=|B|$. Clearly

$$
\|b\|_{L^{p}(w)} \leq C\|a\|_{L^{p}(w)},
$$


giving (4.3). To prove that $b$ satisfies (4.2) we note that for $N_{0} \leq N_{1}$

$$
\tilde{b}_{N_{1}} \geq 2^{N_{1}} \wedge a \geq 2^{N_{0}} \wedge a \text {. }
$$

Let $A_{N}$ be an outer function with modulus $2^{N} \wedge a$. Then $\left|B_{N_{1}}\right| \geq\left|A_{N_{0}}\right|$ a.e. on $\mathbf{T}$. It follows that for $0 \leq r<1$ and $\zeta \in \mathbf{T}$

$$
\left|B_{N_{1}}(r \zeta)\right| \geq\left|A_{N_{0}}(r \zeta)\right| \text {. }
$$

Letting first $N_{1} \rightarrow \infty$ then $r \rightarrow 1$, we get $b \geq 2^{N_{0}} \wedge a$, that yields (4.2).

Now by (4.4) we may assume that for each $n \in Z$ the sequence $\left\{\varphi_{n N}\right\}$ converges to an analytic function $\varphi_{n}$ uniformly on every compact subset of $D$. It is clear that the $\varphi_{n}$ 's are in $H^{\infty}$ and satisfy (4.4). For (4.5) we have

$$
\left|\varphi_{n N}\right|^{1 / 4}\left|B_{N}\right| \leq C 2^{n} \text { a.e. on } \mathbf{T}, \quad \forall n \in \mathbf{Z},
$$

which, together with the subharmonicity of $\left|\varphi_{n N}\right|^{1 / 4}\left|B_{N}\right|$, gives

$$
\left|\varphi_{n N}(r \zeta)\right|^{1 / 4}\left|B_{N}(r \zeta)\right| \leq C 2^{n}, \quad 0 \leq r<1, \zeta \in \mathbf{T} .
$$

Then (4.5) follows by the same limit procedure as above. (4.6) is proved similarly, and (4.7) can easily be derived by passing to the limit as $N \rightarrow \infty$, that can be justified by means of (4.5) and (4.6). Thus the proof of Lemma 4.2 is completed.

Proof of Theorem 4.1. The following result is well known (cf., e.g., [BL]; there the result is stated for Banach spaces, but the proof is easily extendable to the quasi-Banach space setting)

$$
\left(L^{p_{0}}\left(w_{0}\right), L^{p_{1}}\left(w_{1}\right)\right)_{\theta}=L^{p}(w) .
$$

Hence by interpolation

$$
\left(H^{p_{0}}\left(w_{0}\right), H^{p_{1}}\left(w_{1}\right)\right)_{\theta} \subset H^{p}(w) .
$$

So it remains to prove the reverse inclusion. For this, we assume, as in the proof of Theorem 3.1, that $w_{1} \equiv 1, \log w_{0} \in \mathrm{BMO}, 0<p_{0}<\infty$ and $0<p_{1} \leq \infty$. Let then $f \in H^{p}(w)$ with $\|f\|_{H^{p}(w)}<1$. We shall construct a function $F \in$ $\mathscr{F}\left(H^{p_{0}}\left(w_{0}\right), H^{p_{1}}\right)$ such that

$$
\|F(\theta)-f\|_{H^{p}(w)} \leq 1 / 2, \quad\|F\|_{\mathscr{F}\left(H^{p_{0}}\left(w_{0}\right), H^{\left.p_{1}\right)}\right.} \leq C,
$$

with a constant $C$ independent of $f$. An easy iteration argument will then show that $f \in\left(H^{p_{0}}\left(w_{0}\right), H^{p_{1}}\right)_{\theta}$ with $\|f\|_{\theta} \leq C^{\prime}$.

We can find positive functions $g_{0} \in L^{p_{0}}\left(w_{0}\right)$ and $g_{1} \in L^{p_{1}}$ such that

$$
|f| \leq g_{0}^{1-\theta} g_{1}^{\theta} \text {, a.e. on } \mathbf{T} \text { and }\left\|g_{0}\right\|_{L^{p_{0}}\left(w_{0}\right)}<1,\left\|g_{1}\right\|_{L^{p_{1}}}<1 \text {. }
$$

We may evidently assume that also $\log g_{j} \in L^{1} \quad(j=0,1)$. Then let $h_{j}$ be an outer function with modulus $g_{j}(j=0,1)$. Define

$$
f_{0}=\left(f h_{0}^{\theta-1} h_{1}^{-\theta}\right) h_{0}^{1-\theta}, \quad f_{1}=h_{1}^{\theta} .
$$

Clearly, $f=f_{0} f_{1}, f_{0}, f_{1}$ are analytic functions satisfying

$$
\left|f_{0}\right| \leq g_{0}^{1-\theta}, \quad\left|f_{1}\right| \leq g_{1}^{\theta} \text { a.e. on } \mathbf{T} \text {. }
$$


Now applying Lemma 4.2 to $a=g_{0}, p=p_{0}$ and $w=w_{0}$ (resp. $a=g_{1}$, $p=p_{1}$ and $w_{1} \equiv 1$ ) we get $b_{0},\left\{\varphi_{n, 0}\right\}_{n \in \mathbf{Z}}$ (resp. $b_{1},\left\{\varphi_{n, 1}\right\}_{n \in \mathbf{Z}}$ ) as in that lemma. In particular,

$$
\left\|b_{0}\right\|_{L^{p_{0}}\left(w_{0}\right)} \leq C\left\|g_{0}\right\|_{L^{p_{0}}\left(w_{0}\right)} \leq C, \quad\left\|b_{1}\right\|_{L^{p_{1}}} \leq C\left\|g_{1}\right\|_{L^{p_{1}}} \leq C .
$$

Also

$$
f=f_{0} f_{1}=\sum_{n \in \mathbf{Z}} \varphi_{n, 0} f_{0} \sum_{m \in \mathbf{Z}} \varphi_{m, 1} f_{1}
$$

By (4.4)-(4.6)

$$
\begin{aligned}
\sum_{n \in \mathbf{Z}}\left|\varphi_{n, 0} f_{0}\right| & \leq \sum_{n \in \mathbf{Z}}\left|\varphi_{n, 0}\right|^{1 / 2}\left|\varphi_{n, 0}\right|^{1 / 2} b_{0}^{1-\theta} \\
& \leq C \sum_{n \in \mathbf{Z}}\left|\varphi_{n, 0}\right|^{1 / 2} 2^{n} b_{0}^{-\theta} \leq C b_{0}^{1-\theta}
\end{aligned}
$$

Therefore, by the dominated convergence theorem, $\sum_{n \in \mathbf{Z}} \varphi_{n, 0} f_{0}$ converges to $f_{0}$ in $L^{p_{0} /(1-\theta)}\left(w_{0}\right)$. Similarly, $\sum_{n \in Z} \varphi_{n, 1} f_{1}$ converges to $f_{1}$ in $L^{p_{1} / \theta}$ if $p_{1}<$ $\infty$, that we assume for definiteness (if $p_{1}=\infty$, some slight modifications are needed for what follows). Then Hölder inequality implies that there exist $n_{0}, n_{1}$ and $m_{0}, m_{1}$ such that

$$
\left\|f-\sum_{n=n_{0}}^{n_{1}} \varphi_{n, 0} f_{0} \sum_{m=m_{0}}^{m_{1}} \varphi_{m, 1} f_{1}\right\|_{H^{p}(w)} \leq 1 / 2 .
$$

Now for $\zeta \in \mathscr{S}=\{\zeta \in \mathbf{C}: 0<\operatorname{Re} \zeta<1\}$ define

$$
F(\zeta)=\sum_{n=n_{0}}^{n_{1}} \sum_{m=m_{0}}^{m_{1}} 2^{n(\theta-\zeta)} 2^{m(\zeta-\theta)} \varphi_{n, 0} f_{0} \varphi_{m, 1} f_{1} .
$$

Then clearly $F \in \mathscr{F}\left(H^{p_{0}}\left(w_{0}\right), H^{p_{1}}\right)$ and $\|f-F(\theta)\|_{H^{p}(w)} \leq 1 / 2$ by (4.13). It thus remains to dominate $\|F\|_{\mathscr{F}\left(H^{p_{0}}\left(w_{0}\right), H^{p_{1}}\right)}$. We have for $\bar{\eta} \in \mathbf{R}$

$$
|F(i \eta)| \leq \sum_{n=n_{0}}^{n_{1}} 2^{n \theta}\left|\varphi_{n, 0}\right|\left|f_{0}\right| \sum_{m=m_{0}}^{m_{1}} 2^{-m \theta}\left|\varphi_{m, 1}\right|\left|f_{1}\right| .
$$

By (4.4)-(4.6)

$$
\begin{aligned}
& \sum_{m=m_{0}}^{m_{1}} 2^{-m \theta}\left|\varphi_{m, 1}\right|\left|f_{1}\right| \leq \sum_{m=m_{0}}^{m_{1}} 2^{-m \theta}\left|\varphi_{m, 1}\right|^{1 / 2}\left(\left|\varphi_{m, 1}\right|^{1 / 2 \theta} b_{1}\right)^{\theta} \\
& \quad \leq C \sum_{m=m_{0}}^{m_{1}}\left|\varphi_{m, 1}\right|^{1 / 2}=C \sum_{m=m_{0}}^{m_{1}}\left|\varphi_{m, 1}\right|^{1 / 4}\left|\varphi_{m, 1}\right|^{1 / 4} b_{1} b_{1}^{-1} \\
& \quad \leq C \sum_{m=m_{0}}^{m_{1}}\left|\varphi_{m, 1}\right|^{1 / 4} 2^{m} b_{1}^{-1} \leq C
\end{aligned}
$$

Similarly

$$
C \sum_{n=n_{0}}^{n_{1}} 2^{n \theta}\left|\varphi_{n, 0}\right|\left|f_{0}\right| \leq C \sum_{n=n_{0}}^{n_{1}}\left|\varphi_{n, 0}\right|^{1 / 2} 2^{n} \leq C b_{0} .
$$


Combining the previous estimates with the fact that $\left\|b_{0}\right\|_{L^{p_{0}}\left(w_{0}\right)} \leq C$ we get

$$
\|F(i \eta)\|_{L^{p_{0}}\left(w_{0}\right)} \leq C, \quad \forall \eta \in \mathbf{R} .
$$

A similar reasoning shows that $\|F(1+i \eta)\|_{L^{p_{1}}} \leq C(\forall \eta \in \mathbf{R})$. Thus

$$
\|F\|_{\mathscr{F}\left(H^{p_{0}}\left(w_{0}\right), H^{p_{1}}\right)} \leq C,
$$

which concludes the proof of Theorem 4.1.

Remark. We can prove the following refinement of Lemma 4.2.

Let $w$ be a weight such that $\log w \in \mathrm{BMO}$. Then for every positive function $a \in L^{1}(w)$ there exist $b \in L^{1}(w)$ and a sequence $\left\{\varphi_{n}\right\}_{n \in \mathbf{Z}} \subset H^{\infty}$ which satisfy (4.2), (4.4)-(4.7) and the following strengthening of (4.3)

$$
\int_{0}^{t} b^{*}(s) d s \leq \int_{0}^{t} a^{*}(s) d s, \quad \forall t>0,
$$

where $a^{*}$ and $b^{*}$ are respectively the decreasing rearrangements of $a$ and $b$ with respect to the measure $w d m$ on $\mathbf{T}$.

The essential idea of the proof of the above statement is almost the same as that of the proof of Lemma 4.2 (see [X2] for a similar lemma in the nonweighted case). Using this refinement of Lemma 4.2 and arguments of [X2], we can show the following weighted version of the main result of [X2].

Let $1 \leq p_{0}, p_{1} \leq \infty, p_{0} \neq p_{1}$ and $w_{0}, w_{1}$ be weights such that $\log w_{j} \in L^{1}$ $(j=0,1)$. Suppose $\log \left(w_{0}^{1 / p_{0}} w_{1}^{-1 / p_{1}}\right) \in \mathrm{BMO}$. Then for every function $f \in$ $H^{p_{0}}\left(w_{0}\right)+H^{p_{1}}\left(w_{1}\right)$ there exists a linear operator $T$ defined on $L^{p_{0}}\left(w_{0}\right)+L^{p_{1}}\left(w_{1}\right)$ such that $T(f)=f$ which maps $L^{p_{j}}\left(w_{j}\right)$ into $H^{p_{j}}\left(w_{j}\right)(j=0,1)$ and whose norms on these spaces are dominated by a constant depending only on $p_{0}, p_{1}$ and the BMO-norm of $\log \left(w_{0}^{1 / p_{0}} w_{1}^{-1 / p_{1}}\right)$.

This result is much stronger than Theorems 3.1 and 4.1 (in the case where $1 \leq$ $\left.p_{0}, p_{1} \leq \infty, p_{0} \neq p_{1}\right)$. An interesting consequence of it is that with the above hypotheses on $p_{0}, p_{1}$ and $w_{0}, w_{1},\left(H^{p_{0}}\left(w_{0}\right), H^{p_{1}}\left(w_{1}\right)\right)$ is a Calderón-Mitjagin couple. The reader is referred to [X2] for more discussion and consequences of this kind of results.

\section{VeCTOR-VALUEd HARdY SPACES: THE REAL INTERPOLATION}

From now on we shall deal with Hardy spaces of functions with values in quasi-Banach lattices. All quasi-Banach lattices will be those of measurable functions on a measure space $(\Omega, \mu)$. They will be subject to the conditions described in $\S 1$.

Let $w$ be a weight on $\mathbf{T}$ such that $\log w \in L^{1}$. Denote by $\varphi$ the outer function with modulus $w$. For a quasi-Banach lattice $E$ on $(\Omega, \mu)$, the weighted spaces $L^{p}(E, w)$ and $H^{p}(E, w)$ are defined as follows:

$$
\begin{aligned}
L^{p}(E ; w) & =\left\{f: f w^{1 / p} \in L^{p}(E)\right\}=\left\{f: f \varphi^{1 / p} \in L^{p}(E)\right\}, \\
H^{p}(E ; w) & =\left\{f: f \varphi^{1 / p} \in H^{p}(E)\right\} .
\end{aligned}
$$

The quasi-norm on these spaces is given by the functional $f \mapsto\left(\int_{T}\|f\|_{E}^{p} w d m\right)^{1 / p}$. We recall that our $L^{p}(E)$ and $H^{p}(E)$ are slightly "nonstandard" (cf. $\S 1$ ).

We shall use the following well-known result several times: if $X$ is a UMD Banach space and $w$ is a weight in $A_{p} \quad(1<p<\infty)$ then the Hilbert transform 
$H$ is bounded from $\widetilde{L}^{p}(X ; w)$ into itself (the proof of that is the same as in the scalar case, cf. [CF]).

For technical reasons it is convenient to introduce a definition formalizing the property expressed by Lemma 3.3. We begin with the nonweighted case because of its importance.

Definition 5.1. Let $0<p \leq \infty$. A quasi-Banach lattice $E$ of measurable functions on $(\Omega, \mu)$ is said to have $p$-majoration property if there exists $K>0$ such that for every integer $k>K$ the following is true. Given a positive function $a \in L^{p}(E)$, there exists $b \in L^{p}(E)$ satisfying

(5.1) $b \geq a$, a.e. on $\mathbf{T} \times \Omega$;

(5.2) $\|b\|_{L^{p}(E)} \leq C\|a\|_{L^{p}(E)}$

(5.3) $\left|H\left(b(\cdot, \omega)^{1 / 2 k}\right)\right| \leq C b(\cdot, \omega)^{1 / 2 k}$ a.e. on $\mathbf{T} \times \Omega$,

where $C$ is a constant independent of $a$ (it is allowed for $C$ to depend on $k)$.

Note that it is assumed implicitly in $(5.3)$ that $H\left(b(\cdot, \omega)^{1 / 2 k}\right)$ can be interpreted as a bimeasurable function on $T \times \Omega$. The exponent $1 / 2 k$ does not look very natural, but we have taken it for uniformity reasons (compare with Lemma 3.3 and Definition 5.6 below).

Now we give some examples.

Lemma 5.2. If $E^{(\alpha)}$ is $U M D$ for some $\alpha>0$ then $E$ has p-majoration property for $0<p<\infty$. The space $L^{\infty}(\Omega)$ has $p$-majoration property for $0<p \leq \infty$.

This will follow from Lemma 5.7 and Corollary 5.9 below.

Now let $E_{0}, E_{1}$ be quasi-Banach lattices of measurable functions on $(\Omega, \mu)$. Then clearly $\left(H^{p_{0}}\left(E_{0}\right), H^{p_{1}}\left(E_{1}\right)\right)$ is an interpolation couple for $0<p_{0}, p_{1} \leq$ $\infty$. Moreover, $\left(\frac{L^{p_{0}}\left(E_{0}\right)}{H^{p_{0}}\left(E_{0}\right)}, \frac{L^{p_{1}}\left(E_{1}\right)}{H^{p_{1}}\left(E_{1}\right)}\right)$ can also be viewed as an interpolation couple, since the both quotient spaces embed into $\frac{L^{s}\left(L^{s}(\Omega, \varphi d \mu)\right)}{H^{s}\left(L^{s}(\Omega, \varphi d \mu)\right)}$ for some small $s>0$ and some density $\varphi$. So we may consider $K$ - and $J$-functionals for these spaces. The main results of this section are the following two theorems and their weighted counterparts discussed later on.

Theorem 5.3. Let $0<p_{0}<\infty, 0<p_{1} \leq \infty, E_{0}, E_{1}$ be quasi-Banach lattices of measurable functions on $(\Omega, \mu)$. Suppose that $E_{0}^{(\alpha)}$ is a UMD-space for some $\alpha>0$ and $E_{1}$ has $p_{1}$-majoration property. Then for any $t>0$ and any $f \in H^{p_{0}}\left(E_{0}\right)+H^{p_{1}}\left(E_{1}\right)$,

$$
K\left(t, f ; H^{p_{0}}\left(E_{0}\right), H^{p_{1}}\left(E_{1}\right)\right) \leq C K\left(t, f ; L^{p_{0}}\left(E_{0}\right), L^{p_{1}}\left(E_{1}\right)\right),
$$

where $C$ is a constant depending only on $E_{j}$ and $p_{j}(j=0,1)$.

Theorem 5.4. Under the hypotheses of Theorem 5.3, for any $t>0$ and any

$$
\tilde{f} \in \frac{L^{p_{0}}\left(E_{0}\right)}{H^{p_{0}}\left(E_{0}\right)} \cap \frac{L^{p_{1}}\left(E_{1}\right)}{H^{p_{1}}\left(E_{1}\right)}
$$

there exists $f \in L^{p_{0}}\left(E_{0}\right) \cap L^{p_{1}}\left(E_{1}\right)$ generating the class $\tilde{f}$ in the both quotient spaces simultaneously such that

$$
J\left(t, f ; L^{p_{0}}\left(E_{0}\right), L^{p_{1}}\left(E_{1}\right)\right) \leq C J\left(t, \tilde{f} ; \frac{L^{p_{0}}\left(E_{0}\right)}{H^{p_{0}}\left(E_{0}\right)}, \frac{L^{p_{1}}\left(E_{1}\right)}{H^{p_{1}}\left(E_{1}\right)}\right),
$$


where $C$ is a constant depending only on $E_{j}$ and $p_{j}(j=0,1)$.

Remarks (they apply also to Theorems 5.10 and 5.11 below).

(i) Of course, (5.4) and (5.5) can be reversed (with $C=1$ in the reverse inequalities).

(ii) We have already mentioned in $\S 3$ that (5.4) and (5.5) can easily be reduced to each other.

The following corollary is immediate from Theorem 5.3 .

Corollary 5.5. Under the hypotheses of Theorem 5.3, for any $0<\theta<1$ and $0<q \leq \infty$ the space $\left(H^{p_{0}}\left(E_{0}\right), H^{p_{1}}\left(E_{1}\right)\right)_{\theta q}$ coincides (with equivalent quasinorms) with the subspace of $\left(L^{p_{0}}\left(E_{0}\right), L^{p_{1}}\left(E_{1}\right)\right)_{\theta q}$ consisting of the functions analytic in the first variable. In particular,

$$
\left(H^{p_{0}}\left(E_{0}\right), H^{p_{1}}\left(E_{1}\right)\right)_{\theta p}=H^{p}\left(\left(E_{0}, E_{1}\right)_{\theta p}\right),
$$

where $\frac{1}{p}=\frac{1-\theta}{p_{0}}+\frac{\theta}{p_{1}}$.

We shall not give the proofs of Theorems 5.3 and 5.4 separately, because they are nothing but partial cases of analogous results with weights, that we are going to present now. First of all we should define an appropriate majoration property in the weighted case. It turns out that such a property should include a certain uniformity condition.

Definition 5.6. Let $0<p \leq \infty$ and $\mathscr{W}$ be a class of weights on T. A quasi-Banach lattice $E$ of measurable functions on $(\Omega, \mu)$ is said to have $p$ majoration property uniformly with respect to all weights in $\mathscr{W}$ if there exists $K>0$ (depending on $E$ and $p$ ) such that for all integers $k>K$ the following is true. Given a weight $u \in \mathscr{W}$, for every positive function $a \in L^{p}(E)$, there exists $b \in L^{p}(E)$ satisfying (5.1), (5.2) and

$$
\left|H\left(u(\cdot) b(\cdot, w)^{1 / 2 k}\right)\right| \leq C u(\cdot) b(\cdot, \omega)^{1 / 2 k} \quad \text { a.e. on } \mathbf{T} \times \Omega,
$$

where $C$ is a constant independent of $a$.

Remark. $C$ may depend on $u$ (and, of course, on $k$ ). The word "uniformly" refers to the fact that $K$ does not depend on $u \in \mathscr{W}$.

We turn to describe some examples of spaces with the property just defined.

Lemma 5.7. The space $L^{\infty}(\Omega, \mu)$ has $\infty$-majoration property uniformly with respect to all weights $u$ satisfying $|H(u)| \leq C u$, for some constant $C$.

For the proof, simply take $b \equiv\|a\|_{\left.L^{\infty}(\Omega, \mu)\right)}$ for a given $a \in L^{\infty}\left(L^{\infty}(\Omega, \mu)\right)$.

To give less trivial examples, consider two measure spaces $\left(\Omega_{1}, \mu_{1}\right),\left(\Omega_{2}, \mu_{2}\right)$ and let $F$ be a quasi-Banach lattice of measurable functions on $\left(\Omega_{1}, \mu_{1}\right)$. Denote by $(\Omega, \mu)$ the product space $\left(\Omega_{1} \times \Omega_{2}, \mu_{1} \times \mu_{2}\right)$ and consider the quasi-Banach lattice $E=F\left(L^{\infty}\left(\Omega_{2}, \mu_{2}\right)\right)$ on $(\Omega, \mu)$ which consists of the bi-measurable functions $g$ on $(\Omega, \mu)$ such that $g\left(\omega_{1}, \cdot\right) \in L^{\infty}\left(\mu_{2}\right)$ for a.e. $\omega_{1} \in \Omega_{1}$ and the function $\omega_{1} \mapsto\left\|g\left(\omega_{1}, \cdot\right)\right\|_{L^{\infty}\left(\mu_{2}\right)}$ is in $F$. The quasi-norm of $g$ in $E$ is then defined as that of this latter function in $F$.

Lemma 5.8. Let $0<p<\infty$. If $F^{(\alpha)}$ is UMD for some $\alpha>0$ then $E$ has $p$ majoration property uniformly with respect to all weights $u$ satisfying $M\left(u^{2}\right) \leq$ $C u^{2}$, for some constant $C$.

Corollary 5.9. Let $E$ be a quasi-Banach lattice of measurable functions on some measure space $(\Omega, \mu)$. Suppose that either $E^{(\alpha)}$ is $U M D$ for some $\alpha>0$ or $E=$ 
$L^{\infty}(\Omega)$. Then for every $0<p<\infty, E$ has p-majorization property uniformly with respect to all weights $u$ satisfying $M\left(u^{2}\right) \leq C u^{2}$, for some constant $C$.

To see this, take in Lemma 5.8 the one-point space either for $\left(\Omega_{2}, \mu_{2}\right)$ or for $\left(\Omega_{1}, \mu_{1}\right)$.

Finally, we remark that Lemma 5.2 follows from Lemma 5.7 and Corollary 5.9 since $H(1)=0$ and $M(1)=1$.

Proof of Lemma 5.8. Take $K$ so large that $K p \geq 1$ and $F^{(2 K)}$ is a UMD-space. Let $k>K$ be an integer and let a weight $u$ satisfy $M\left(u^{2}\right) \leq C u^{2}$. Any positive function $a \in L^{p}(E)$ can be viewed as a measurable function on $\mathbf{T} \times \Omega_{1} \times \Omega_{2}$. We shall construct a majorant $b$ satisfying (5.1), (5.2) and (5.6) that will not actually depend on $\omega_{2} \in \Omega_{2}$.

Set

$$
a_{0}\left(\zeta, \omega_{1}\right)=\operatorname{ess} \sup _{\omega_{2} \in \Omega_{2}}\left|a\left(\zeta, \omega_{1}, \omega_{2}\right)\right|^{1 / 2 k},
$$

and let inductively $a_{n}\left(\cdot, \omega_{1}\right)=u(\cdot)^{-1}\left|H\left(u(\cdot) a_{n-1}\left(\cdot, \omega_{1}\right)\right)\right|(n \geq 1)$. We have $F^{(2 k)} \in \mathrm{UMD}, u^{-2 k p}=\left(u^{2}\right)^{-k p} \in A_{1+k p}$ and $2 k p>1+k p$. Therefore $H$ is bounded from $L^{2 k p}\left(F^{(2 k)} ; u^{-2 k p}\right)$ into itself. Let $C_{1}$ be its norm. Then for $n \geq 0$.

$$
\begin{aligned}
\left\|a_{n}\right\|_{L^{2 k p}\left(E^{(2 k))}\right.} & =\left\|a_{n}\right\|_{L^{2 k p}\left(F^{(2 k))}\right.}=\left\|H\left(u a_{n-1}\right)\right\|_{L^{2 k p}\left(F^{(2 k)} ; u^{-2 k p}\right)} \\
& \leq C_{1}\left\|u a_{n-1}\right\|_{L^{2 k p}\left(F^{(2 k)} ; u^{-2 k p}\right)}=C_{1}\left\|a_{n-1}\right\|_{L^{2 k p}\left(E^{(2 k)}\right)} \\
& \leq \cdots \leq C_{1}^{n}\left\|a_{0}\right\|_{L^{2 k p}\left(E^{(2 k)}\right)}=C_{1}^{n}\|a\|_{L^{p}(E)}^{1 / 2 k} .
\end{aligned}
$$

Choose $C>C_{1}$ in an appropriate way (note that we do not assume that $E^{(2 k)}$ has been renormed to become a Banach space). Then we easily see that the function $b$ defined by $b=\left(\sum_{n \geq 0} C^{-n} a_{n}\right)^{2 k}$ satisfies the desired properties.

Now we are in a position to state the weighted counterparts of Theorems 5.3 and 5.4. Consider the class of weights $\mathscr{B}=\{u: \exists C,|H(u)| \leq C u$ and $\left.M\left(u^{2}\right) \leq C u^{2}\right\}$.

Theorem 5.10. Let $0<p_{0}<\infty, 0<p_{1} \leq \infty, E_{0}$ and $E_{1}$ be quasi-Banach lattices of measurable functions on $(\Omega, \mu)$. Suppose that $E_{0}^{(\alpha)}$ is a UMD-space for some $\alpha>0$ and $E_{1}$ has $p_{1}$-majoration property uniformly for the weights in $\mathscr{B}$. Then for any couple of weights $w_{0}, w_{1}$ with $\log \left(w_{0}^{1 / p_{0}} w_{1}^{-1 / p_{1}}\right) \in \mathrm{BMO}$ we have

$$
\begin{aligned}
& K\left(t, f ; H^{p_{0}}\left(E_{0} ; w_{0}\right), H^{p_{1}}\left(E_{1} ; w_{1}\right)\right) \\
& \quad \leq C K\left(t, f ; L^{p_{0}}\left(E_{0} ; w_{0}\right), L^{p_{1}}\left(E_{1} ; w_{1}\right)\right)
\end{aligned}
$$

for any $f \in H^{p_{0}}\left(E_{0} ; w_{0}\right)+H^{p_{1}}\left(E_{1} ; w_{1}\right)$ and any $t>0$, where the constant $C$ does not depend on $f$ and $t$.

Theorem 5.11. Under the hypotheses of Theorem 5.10, for any $t>0$ and any

$$
\tilde{f} \in \frac{L^{p_{0}}\left(E_{0} ; w_{0}\right)}{H^{p_{0}}\left(E_{0} ; w_{0}\right)} \cap \frac{L^{p_{1}}\left(E_{1} ; w_{1}\right)}{H^{p_{1}}\left(E_{1} ; w_{1}\right)}
$$

there exists $f \in L^{p_{0}}\left(E_{0} ; w_{0}\right) \cap L^{p_{1}}\left(E_{1} ; w_{1}\right)$ generating the class $\tilde{f}$ in the both 
quotient spaces simultaneously such that

$$
\begin{aligned}
& J\left(t, f ; L^{p_{0}}\left(E_{0} ; w_{0}\right), L^{p_{1}}\left(E_{1} ; w_{1}\right)\right) \\
& \quad \leq C J\left(t, \tilde{f} ; \frac{L^{p_{0}}\left(E_{0} ; w_{0}\right)}{H^{p_{0}}\left(E_{0} ; w_{0}\right)}, \frac{L^{p_{1}}\left(E_{1} ; w_{1}\right)}{H^{p_{1}}\left(E_{1} ; w_{1}\right)}\right),
\end{aligned}
$$

where $C$ is a constant independent of $\tilde{f}$ and $t$.

We leave to the reader formulating the counterpart of Corollary 5.5 for the weighted case.

Turning to the proofs of Theorems 5.10 and 5.11, we first multiply all the spaces considered by an outer function with modulus $w_{1}^{1 / p_{1}}$. Thus we can, and do assume that $w_{1} \equiv 1, \log w_{0} \in \mathrm{BMO}$. The following crucial lemma is similar to Lemma 3.4.

Lemma 5.12. Under the hypotheses of Theorem 5.10 , given $f_{0} \in L^{p_{0}}\left(E_{0} ; w_{0}\right)$ and $f_{1} \in L^{p_{1}}\left(E_{1}\right)$, there exists a function $G \in H^{\infty}\left(L^{\infty}(\Omega, \mu)\right)$ such that

$$
\begin{aligned}
& \max \left\{\left\|(1-G) f_{0}\right\|_{L^{p_{0}}\left(E_{0} ; w_{0}\right)},\left\|(1-G) f_{1}\right\|_{L^{p_{0}}\left(E_{0} ; w_{0}\right)}\right\} \\
& \quad \leq C\left\|f_{0}\right\|_{L^{p_{0}}\left(E_{0} ; w_{0}\right)} ; \\
& \max \left\{\left\|G f_{0}\right\|_{L^{p_{1}}\left(E_{1}\right)},\left\|G f_{1}\right\|_{L^{p_{1}}\left(E_{1}\right)}\right\} \leq C\left\|f_{1}\right\|_{L^{p_{1}}\left(E_{1}\right)},
\end{aligned}
$$

where $C$ is independent of $f_{0}, f_{1}$.

Proor. Let $K>0$ be as in Definition 5.6 with $E_{1}$ and $p_{1}$ instead of $E$ and p. Take a large integer $k>K$ such that $k p_{0} \geq 1$ and $E_{0}^{(2 k)}$ is UMD. As in $\S 3$, we find, via Lemma 1.1 , a representation $w_{0}=\left(u_{0} u_{1}^{-1}\right)^{2 k p_{0}}$ where the weights $u_{0}$ and $u_{1}$ satisfy (3.2) and (3.3). (Recall that we also need for this $k p_{0} \geq 2 \gamma$, where $\gamma$ is determined by $w_{0}$.) In particular, $u_{0} \in \mathscr{B}$. Thus, given $a \in L^{p_{1}}\left(E_{1}\right)$, we can find $b$ satisfying (5.1), (5.2) and (5.6) with $u=u_{0}$ and $E, p$ replaced by $E_{1}, p_{1}$.

We are going to apply that to the function $a$ constructed as follows. Let $\Omega_{0} \subset \Omega$ be the support of the space $E_{1}$. Since we have supposed $(\Omega, \mu) \sigma$ finite, there exists a function $\beta \in E_{1}$ strictly positive on $\Omega_{0}$, and all functions in $E_{1}$ vanish off $\Omega_{0}$. We take $a=\left|f_{1}\right|+\varepsilon \beta, \varepsilon$ being a small positive number such that $\|a\|_{L^{1}\left(E_{1}\right)} \leq 2\left\|f_{1}\right\|_{L^{p_{1}\left(E_{1}\right)} \text {. }}$.

With the help of the above majorant $b$ for this $a$, we define a function $\alpha$ on $\mathbf{T} \times \Omega$ by

$$
\alpha=\max \left\{1,\left|f_{0} b^{-1}\right|^{1 / 2 k}\right\} \text { on } \mathbf{T} \times \Omega_{0} ; \quad \alpha=0 \text { on } \mathbf{T} \times\left(\Omega \backslash \Omega_{0}\right) .
$$

Then set (with the convention $0 / 0=0$ )

$$
F=\frac{u_{0} b^{1 / 2 k}+i H\left(u_{0} b^{1 / 2 k}\right)}{u_{0} b^{1 / 2 k} \alpha+i H\left(u_{0} b^{1 / 2 k} \alpha\right)}, \quad G=1-\left(1-F^{2 k}\right)^{2 k} .
$$

Of course, the operator $H$ is applied here in the first variable. Clearly, $F=0$ on $\mathbf{T} \times\left(\Omega \backslash \Omega_{0}\right)$ and by $(5.1)$ for a.e. $\omega \in \Omega_{0}$ the function $b(\cdot, \omega) \alpha(\cdot, \omega)$ is bounded away from zero. Hence $F$ is analytic in the first variable. Moreover, by (5.6)

$$
|F| \leq(1+C) \frac{u_{0} b^{1 / 2 k}}{u_{0} b^{1 / 2 k} \alpha} \leq \frac{1+C}{\alpha} \leq 1+C \quad \text { a.e. on } \mathbf{T} \times \Omega_{0}
$$


Therefore, $F \in H^{\infty}\left(L^{\infty}(\Omega)\right)$. Consequently, $G \in H^{\infty}\left(L^{\infty}(\Omega)\right)$ and $G=0$ a.e. on $T \times\left(\Omega \backslash \Omega_{0}\right)$,

$$
|G| \leq C|F|^{2 k} \leq C / \alpha^{2 k} \quad \text { a.e. on } \mathbf{T} \times \Omega_{0} .
$$

It follows, in particular, that $\|G\|_{H^{\infty}\left(L^{\infty}(\Omega)\right)} \leq C$. Hence to prove (5.9) and (5.10) it remains to show

$$
\left\|G f_{0}\right\|_{L^{p_{1}\left(E_{1}\right)}} \leq C\left\|f_{1}\right\|_{L^{p_{1}\left(E_{1}\right)}}, \quad\left\|(1-G) f_{1}\right\|_{L^{p_{0}}\left(E_{0} ; w_{0}\right)} \leq C\left\|f_{0}\right\|_{L^{p_{0}}\left(E_{0} ; w_{0}\right)} .
$$

The first inequality follows immediately from

$$
\left|G f_{0}\right| \leq C b \text { and }\|b\|_{L^{p_{1}\left(E_{1}\right)}} \leq C\|a\|_{L^{p_{1}\left(E_{1}\right)}} \leq C\left\|f_{1}\right\|_{L^{p_{1}\left(E_{1}\right)}} .
$$

To prove the second inequality note that

$$
\begin{aligned}
|1-G| & \leq C|1-F|^{2 k} \\
& \leq C\left[(\alpha-1)^{2 k}+u_{0}^{-2 k} b^{-1}\left|H\left(u_{0} b^{1 / 2 k}(\alpha-1)\right)\right|^{2 k}\right], \quad \text { on } \mathbf{T} \times \Omega_{0} .
\end{aligned}
$$

Hence (recall that $f_{1}=0$ on $\mathbf{T} \times\left(\Omega \backslash \Omega_{0}\right)$ )

$$
\begin{aligned}
& \left\|(1-G) f_{1}\right\|_{L^{p_{0}}\left(E_{0}, w_{0}\right)} \\
& \quad \leq C\left[\left\|(\alpha-1)^{2 k} b\right\|_{L^{p_{0}}\left(E_{0} ; w_{0}\right)}+\left\|H\left(u_{0} b^{1 / 2 k}(\alpha-1)\right)\right\|_{L^{2 k p_{0}}\left(E_{0}^{(2 k)} ; u_{1}^{-2 k p_{0}}\right)}\right] .
\end{aligned}
$$

Now $E_{0}^{(2 k)}$ is UMD, $2 k p_{0} \geq 1+k p_{0}$ and $u_{1}^{-2 k p_{0}} \in A_{1+k p_{0}}$. Therefore $H$ is bounded on $L^{2 k p_{0}}\left(E_{0}^{(2 k)} ; u_{1}^{-2 k p_{0}}\right)$, and it follows that the second summand on the right in the above inequality is dominated by the first one. Now $(\alpha-1)^{2 k} b \leq$ $\left|f_{0}\right|$ on $\mathbf{T} \times \Omega_{0}$. Combining all the above estimates, we obtain

$$
\left\|(1-G) f_{1}\right\|_{L^{p_{0}}\left(E_{0} ; w_{0}\right)} \leq C\left\|f_{0}\right\|_{L^{p_{0}}\left(E_{0} ; w_{0}\right)},
$$

which concludes the proof of Lemma 5.12.

Now Theorems 5.10 and 5.11 follow form Lemma 5.12 by the same reasoning. We begin with the first of them. Let $f_{0} \in L^{p_{0}}\left(E_{0} ; w_{0}\right), f_{1} \in L^{p_{1}}\left(E_{1}\right)$ with $f=f_{0}+f_{1}$ and

$$
\left\|f_{0}\right\|_{L^{p_{0}}\left(E_{0} ; w_{0}\right)}+t\left\|f_{1}\right\|_{L^{p_{1}}\left(E_{1}\right)} \leq 2 K\left(t, f ; L^{p_{0}}\left(E_{0} ; w_{0}\right), L^{p_{1}}\left(E_{1}\right)\right) .
$$

Then find a function $G \in H^{\infty}\left(L^{\infty}(\Omega)\right)$ such that (5.9), (5.10) hold. Set $g=$ $(1-G) f, h=G f$. Then we clearly have

$$
\|g\|_{L^{p_{0}\left(E_{0} ; w_{0}\right)}} \leq C\left\|f_{0}\right\|_{L^{p_{0}\left(E_{0} ; w_{0}\right)}} \text { and }\|h\|_{L^{p_{1}\left(E_{1}\right)}} \leq C\left\|f_{1}\right\|_{L^{p_{1}\left(E_{1}\right)}} .
$$

On the other hand $f=g+h$ and $g, h$ are analytic in the first variable (because $f$ and $G$ are). Thus $g \in H^{p_{0}}\left(E_{0} ; w_{0}\right), h \in H^{p_{1}}\left(E_{1}\right)$ and (5.7) clearly follows from

$$
K\left(t, f ; H^{p_{0}}\left(E_{0}, w_{0}\right), H^{p_{1}}\left(E_{1}\right)\right) \leq\|g\|_{H^{p_{0}}\left(E_{0} ; w_{0}\right)}+t\|h\|_{H^{p_{1}}\left(E_{1}\right)} .
$$

To prove Theorem 5.11 take

such that

$$
\tilde{f} \in \frac{L^{p_{0}}\left(E_{0} ; w_{0}\right)}{H^{p_{0}}\left(E_{0} ; w_{0}\right)} \cap \frac{L^{p_{1}}\left(E_{1}\right)}{H^{p_{1}}\left(E_{1}\right)}
$$

$$
J\left(t, \tilde{f} ; \frac{L^{p_{0}}\left(E_{0} ; w_{0}\right)}{H^{p_{0}}\left(E_{0} ; w_{0}\right)}, \frac{L^{p_{1}}\left(E_{1}\right)}{H^{p_{1}}\left(E_{1}\right)}\right)<1
$$


Then there exist $f_{0} \in L^{p_{0}}\left(E_{0} ; w_{0}\right)$ and $f_{1} \in L^{p_{1}}\left(E_{1}\right)$ representing the class $\tilde{f}$ such that $\left\|f_{0}\right\|_{L^{p_{0}}\left(E_{0} ; w_{0}\right)}<1,\left\|f_{1}\right\|_{L^{p_{1}}\left(E_{1}\right)}<t^{-1}$. Again apply Lemma 5.12 to get $G \in H^{\infty}\left(L^{\infty}(\Omega)\right)$. Then set $f=G f_{0}+(1-G) f_{1}$. By (5.9) and (5.10)

$$
\|f\|_{L^{p_{0}\left(E_{0} ; w_{0}\right)}} \leq C \text { and }\|f\|_{L^{p_{1}\left(E_{1}\right)}} \leq C t^{-1},
$$

implying $J\left(t, f ; L^{p_{0}}\left(E_{0} ; w_{0}\right), L^{p_{1}}(E)\right) \leq C$. On the other hand, $f$ generates the same class $\tilde{f}$, because $G$ is analytic in the first variable. This completes the proof of Theorem 5.11 .

Theorems 5.3 and 5.4 can be proved similarly. One needs just to note that if $w_{0}=w_{1} \equiv 1$, then clearly one can take $u_{0}=u_{1} \equiv 1$ in the above factorization.

\section{Vector-VAlued HaRdy SPACES: THE COMPLEX INTERPOLATION}

In this section we give counterparts of the results of $\S 4$ for the spaces of vector-valued functions. It is convenient to introduce a notion inspired by Lemma 4.2.

Definition 6.1. Let $0<p \leq \infty, E$ be a quasi-Banach lattice of measurable functions on $(\Omega, \mu)$ and $w$ a weight on $\mathbf{T}$. The space $L^{p}(E ; w)$ is said to admit sufficiently many analytic decompositions of unity if for every function $a$ in a dense subset of $L^{p}(E ; w)$ there exist $b \in L^{p}(E ; w)$ and a sequence $\left\{\varphi_{n}\right\}_{n \in \mathbf{Z}}$ in $H^{\infty}\left(L^{\infty}(\Omega, \mu)\right)$ satisfying the following properties:

(6.1) $b \geq|a|$, a.e. on $T \times \Omega$;

(6.2) $\|b\|_{L^{p}(E ; w)} \leq C\|a\|_{L^{p}(E ; w)}$;

(6.3) $\left\|\varphi_{n}\right\|_{H^{\infty}\left(L^{\infty}(\Omega)\right)} \leq C, \forall n \in \mathbf{Z}$;

(6.4) $\left|\varphi_{n}\right|^{1 / 4} b \leq C 2^{n}$, a.e. on $\mathbf{T} \times \Omega, \forall n \in \mathbf{Z}$;

(6.5) $\sum_{n \in \mathbf{Z}}\left|\varphi_{n}\right|^{1 / 4} 2^{n} \leq C b$, a.e. on $\mathbf{T} \times \Omega$;

(6.6) $\sum_{n \in \mathbf{Z}} \varphi_{n}=1$, a.e. on $\mathbf{T} \times \Omega$;

(6.7) $\sum_{n \in \mathbf{Z}} \varphi_{n} a$ converges to $a$ in $L^{p}(E ; w)$,

where $C$ is a constant depending on $E, p$ and $w$ only.

Again (as in $\S 4)$ we note that the $\varphi_{n}$ 's behave roughly as the functions $\chi_{\left\{2^{n-1}<b \leq 2^{n}\right\}}$.

It turns out that $L^{p}(E ; w)$ admits sufficiently many analytic decompositions of unity if $E$ is the same as in Lemma 5.8.

Lemma 6.2. Let $\left(\Omega_{1}, \mu_{1}\right),\left(\Omega_{2}, \mu_{2}\right)$ be two measure spaces and $F$ a quasiBanach lattice of measurable functions on $\left(\Omega_{1}, \mu_{1}\right)$. Let $E=F\left(L^{\infty}\left(\Omega_{2}, \mu_{2}\right)\right)$ be the quasi-Banach lattice of measurable functions on $(\Omega, \mu)=\left(\Omega_{1} \times \Omega_{2}, \mu_{1} \times\right.$ $\left.\mu_{2}\right)$ defined as in $\S 5$. If $F^{(\alpha)}$ is UMD for some $\alpha>0$, and $0<p<\infty$ then $L^{p}(E ; w)$ admits sufficiently many analytic decompositions of unity for every weight $w$ such that $\log w \in \mathrm{BMO}$.

Remark. Of course, under the same hypotheses $L^{p}(F ; w)$ and $L^{p}\left(L^{\infty}\left(\Omega_{2}\right) ; w\right)$ also admit sufficiently many analytic decompositions of unity (take the onepoint space either for $\left(\Omega_{2}, \mu_{2}\right)$ or for $\left.\left(\Omega_{1}, \mu_{1}\right)\right)$. It can be shown that, moreover, $L^{\infty}\left(L^{\infty}\left(\Omega_{2}\right)\right)$ possesses all the properties described in Definition 6.1 except (6.7) (modify the reasoning below just as it has been done in the proof of Lemma 4.2 for $p=\infty)$.

Proof of Lemma 6.2. It is similar to the proof of Lemma 4.2. We first claim that the family of bounded functions on $\mathrm{T} \times \Omega$ is dense in $L^{p}(E ; w)$. Indeed, 
let $f \in L^{p}(E ; w)$. This is a function in three variables $\left(\zeta, \omega_{1}, \omega_{2}\right)$ on $\mathbf{T} \times$ $\Omega_{1} \times \Omega_{2}$. Set $E_{n}=\left\{\left(\zeta, \omega_{1}\right) \in \mathbf{T} \times \Omega_{1}\right.$ : ess $\left.\sup _{\Omega_{2}}\left|f\left(\zeta, \omega_{1}, \omega_{2}\right)\right| \leq n\right\}$ and $f_{n}=f \chi_{E_{n} \times \Omega_{2}} \quad(n \in \mathbf{N})$. Then for every $n \in \mathbf{N} f_{n}$ is bounded on $\mathbf{T} \times \Omega$. It is clear that the UMD condition on $F^{(\alpha)}$ implies that the dominated convergence theorem is valid in $F$. Hence, it is also valid in $L^{p}(F ; w)$. Then we easily deduce that $f_{n}$ converges to $f$ in $L^{p}(E ; w)$. This proves our claim.

Now take a large $k$ so that $k p \geq 1, F^{(2 k)}$ is UMD and $w$ admits a factorization $w=\left(u_{0} u_{1}^{-1}\right)^{2 k p}$ with $u_{0}, u_{1}$ satisfying (3.2), (3.3). Let $a \in L^{p}(E ; w)$ be a bounded function on $\mathbf{T} \times \Omega$. Assume also $a$ positive. We shall construct functions $b$ and $\varphi_{n}(n \in \mathbf{Z})$ satisfying (6.1)-(6.7) which will not depend on $\omega_{2}$.

Since $a$ is bounded and positive, $a<2^{N}$ a.e. on $\mathbf{T} \times \Omega$ for some integer $N$. As in the proof of Lemma 4.2, we define by induction two sequences $\left\{G_{n}\right\}_{n \leq N} \subset H^{\infty}\left(L^{\infty}\left(\Omega_{1}\right)\right)$ and $\left\{b_{n}\right\}_{n \leq N} \subset L^{\infty}\left(L^{\infty}\left(\Omega_{1}\right)\right)$ as follows:

$$
G_{N} \equiv 1, \quad b_{N}\left(\zeta, \omega_{1}\right)=\text { ess } \sup _{\Omega_{2}} a\left(\zeta, \omega_{1}, \omega_{2}\right)
$$

and for $n \leq N-1$

$$
\begin{aligned}
& \alpha_{n}\left(\zeta, \omega_{1}\right)=\max \left\{1,\left(2^{-n} b_{n+1}\left(\zeta, \omega_{1}\right)\right)^{1 / 4 k}\right\}, \\
& F_{n}=\frac{u_{0}+i H u_{0}}{\alpha_{n} u_{0}+i H\left(\alpha_{n} u_{0}\right)} \quad(H \text { is applied in the variable } \zeta \text { here }) \\
& G_{n}=1-\left(1-F_{n}^{16 k}\right)^{8 k}, \quad b_{n}=b_{n+1}+\delta\left|G_{n+1}-G_{n}\right|^{1 / 4} 2^{n+1},
\end{aligned}
$$

where $\delta \in(0,1)$ is a constant to be determined later.

It follows from (3.2) that $F_{n}, G_{n}$ are in $H^{\infty}\left(L^{\infty}(\Omega)\right)$ and, moreover,

$$
\begin{gathered}
\left|F_{n}\right| \leq C \alpha_{n}^{-1} \leq C, \\
\left|G_{n}\right| \leq C\left|F_{n}\right|^{16 k} \leq C \alpha_{n}^{-16 k} \leq C, \quad \text { a.e. on } \mathbf{T} \times \Omega .
\end{gathered}
$$

Let $\varphi_{n}=G_{n}-G_{n-1} \quad(n \leq N)$. Then $\varphi_{n} \in H^{\infty}\left(L^{\infty}(\Omega)\right)$ (in fact, $\varphi_{n}$ does not depend on $\omega_{2}$ ) and (6.3) is true. Letting

$$
\lim _{n \rightarrow-\infty} b_{n}=b \quad(\text { a.e. on } \mathbf{T} \times \Omega),
$$

we easily check that

$$
a \leq b \leq b_{n}+C 2^{n}, \quad \forall n \leq N,
$$

that shows (6.1). The proofs of (6.4) and (6.6) are similar to the corresponding parts of the proof of Lemma 4.2. We are going to show (6.2). Fix $n \leq N$. We have

$$
\begin{aligned}
\left\|b_{n}\right\|_{L^{p}(E ; w)} \leq & C\|a\|_{L^{p}(E ; w)}+C \delta\left\|\sum_{m=n}^{N-1}\left|\alpha_{m}-1\right|^{2 k} 2^{m}\right\|_{L^{p}(E ; w)} \\
& +C \delta\left\|\sum_{m=n}^{N-1}\left|H\left(u_{0}\left(\alpha_{m}-1\right)\right)\right|^{2 k} 2^{m} u_{0}^{-2 k}\right\|_{L^{p}(E ; w)} .
\end{aligned}
$$

Now for $r>0$ we denote by $E\left(l^{r}\right)$ the quasi-Banach lattice of all sequences $\left\{x_{n}\right\}$ of functions in $E$ such that $\omega \mapsto\left(\sum_{n}\left|x_{n}(\omega)\right|^{r}\right)^{1 / r}$ is in $E$, with the natural quasi-norm. Since $2 k>1$ and $F^{(2 k)}$ is UMD, it follows that $F^{(2 k)}\left(l^{2 k}\right)$ 
is also UMD (cf. [RF]). Hence (recall that $u_{1}^{-2 k p} \in A_{1+k p}$ and $2 k p \geq 1+k p>$ 1)

$$
\begin{gathered}
\left\|\sum_{m=n}^{N-1}\left|H\left(u_{0}\left(\alpha_{m}-1\right)\right)\right|^{2 k} 2^{m} u_{0}^{-2 k}\right\|_{L^{p}(E ; w)} \\
=\left\|\left\{H\left(2^{m / 2 k} u_{0}\left(\alpha_{m}-1\right)\right)\right\}_{m=n}^{N-1}\right\|_{L^{2 k p}\left(F^{(2 k)}\left(l^{2 k}\right) ; u_{1}^{-2 k p}\right)}^{2 k} \\
\leq C\left\|\left\{2^{m / 2 k} u_{0}\left(\alpha_{m}-1\right)\right\}_{m=n}^{N-1}\right\|_{L^{2 k p}\left(F^{(2 k)}\left(l^{2 k}\right) ; u_{1}^{-2 k p}\right)} \\
=C\left\|\sum_{m=n}^{N-1}\left(\alpha_{m}-1\right)^{2 k} 2^{m}\right\|_{L^{p}(E ; w)} .
\end{gathered}
$$

On the other hand, as in the proof of Lemma 4.2, we may show that

$$
\sum_{m=n}^{N-1}\left|\alpha_{m}-1\right|^{2 k} 2^{m} \leq C b_{n}
$$

Combining all the inequalities obtained, we get

$$
\left\|b_{n}\right\|_{L^{p}(E ; w)} \leq C\|a\|_{L^{p}(E ; w)}+C \delta\left\|b_{n}\right\|_{L^{p}(E ; w)} .
$$

Taking $\delta=(2 C)^{-1}$, we get finally

$$
\left\|b_{n}\right\|_{L^{p}(w)} \leq C\|a\|_{L^{p}(w)}, \quad \forall n \leq N-1,
$$

which yields (6.2) by choosing $\delta=(2 C)^{-1}$ and letting $n \rightarrow-\infty$. (4.6) holds with $C=\delta^{-1}$. Finally, (6.7) follows form (6.2), (6.4), (6.5) and the dominated convergence theorem in $L^{p}(F ; w)$. This finishes the proof of Lemma 6.2.

We recall that a quasi-Banach lattice $E$ is said to be $\sigma$-order continuous if for every decreasing sequence $\left\{x_{n}\right\}_{n \geq 0}$ of positive functions in $E \wedge_{n \geq 0} x_{n}=0$ implies $\lim _{n \rightarrow \infty}\left\|x_{n}\right\|=0$.

Theorem 6.3. Let $0<p_{0}, p_{1} \leq \infty, w_{0}, w_{1}$ be weights such that $\log w_{j} \in L^{1}$ $(j=0,1)$ and $E_{0}$ and $E_{1}$ be quasi-Banach lattices of measurable functions on $(\Omega, \mu)$ such that $L^{p_{j}}\left(E_{j} ; w_{j}\right)(j=0,1)$ admits sufficiently many analytic decompositions of unity. If one of $E_{0}, E_{1}$ is $\sigma$-order continuous, then for every $0<\theta<1$

$$
\left(H^{p_{0}}\left(E_{0} ; w_{0}\right), H^{p_{1}}\left(E_{1} ; w_{1}\right)\right)_{\theta}=H^{p}\left(\left(E_{0}, E_{1}\right)_{\theta} ; w\right),
$$

where $\frac{1}{p}=\frac{1-\theta}{p_{0}}+\frac{\theta}{p_{1}}$ and $w=w_{0}^{p(1-\theta) / p_{0}} w_{1}^{p_{\theta} / p_{1}}$.

Remark. If we impose our usual condition $\log \left(w_{0}^{1 / p_{0}} w_{1}^{-1 / p_{1}}\right) \in$ BMQ, we deduce that (6.8) does hold if $E_{0}$ and $E_{1}$ are as $E$ in Lemma 6.2. (To do this, we should multiply all the spaces involved by an outer function with modulus equal to $w_{1}^{1 / p_{1}}$ and then apply Theorem 6.3 to the couple of weights $\left(w_{0}^{1 / p_{0}} w_{1}^{-1 / p_{1}}, 1\right)$, instead of $\left(w_{0}, w_{1}\right)$.)

Proof. Let $E=\left(E_{0}, E_{1}\right)_{\theta}$. We need only to verify the inclusion

$$
H^{p}(E ; w) \subset\left(H^{p_{0}}\left(E_{0} ; w_{0}\right), H^{p_{1}}\left(E_{1} ; w_{1}\right)\right)_{\theta} .
$$


Let then $f \in H^{p}(E ; w)$ with quasi-norm less than 1 . We shall construct a function $F \in \mathscr{F}\left(H^{p_{0}}\left(E_{0} ; w_{0}\right), H^{p_{1}}\left(E_{1} ; w_{1}\right)\right)$ such that

$$
\|F(\theta)-f\|_{H^{p}(E ; w)} \leq \varepsilon, \quad \text { and }\|F\|_{\mathscr{F}\left(H^{p_{0}}\left(E_{0} ; w_{0}\right), H^{p_{1}}\left(E_{1} ; w_{1}\right)\right)} \leq C,
$$

with a constant $C$ independent of $f$. Then we fix $\varepsilon$ small enough (its magnitude depends on the constant in triangle inequality) and iterate, that will prove the desired inclusion.

Choose positive functions $g_{j} \in L^{p_{j}}\left(E_{j} ; w_{j}\right)(j=0,1)$ such that

$$
|f| \leq g_{0}^{1-\theta} g_{1}^{\theta} \text {, a.e. on } \mathbf{T} \times \Omega \text { and }\left\|g_{j}\right\|_{L^{p_{j}}\left(E_{j} ; w_{j}\right)}<1, \quad j=0,1 \text {. }
$$

We may clearly assume that $g_{j}$ belongs to the dense subset of $L^{p_{j}}\left(E_{j} ; w_{j}\right)$ in the Definition 6.1 and that $\log g_{j}(\cdot, w) \in L^{1}$ for a.e. $\omega \in \Omega(j=0,1)$. Then as in the proof of Theorem 4.1 we have two functions $f_{j}(j=0,1)$ analytic in the first variable such that

$$
f=f_{0} f_{1},\left|f_{0}\right| \leq g_{0}^{1-\theta},\left|f_{1}\right| \leq g_{1}^{\theta} \text { a.e. on } \mathbf{T} \times \Omega .
$$

By the hypotheses, there exist $b_{j}$ and $\left\{\varphi_{n, j}\right\}_{n \in Z}$ such that (6.1)-(6.7) hold with $E, p, a, w$ replaced, respectively, by $E_{j}, p_{j}, g_{j}, w_{j}(j=0,1)$. In particular, $\sum_{n \in Z} \varphi_{n, j} g_{j}$ converges to $g_{j}$ in $L^{p_{j}}\left(E_{j} ; w_{j}\right)$. By Hölder inequality and (6.3)$(6.5)$

$$
\begin{aligned}
& \sum_{n \in \mathbf{Z}}\left|\varphi_{n, 0}\right|\left|f_{0}\right| \leq \sum_{n \in \mathbf{Z}}\left|\varphi_{n, 0}\right| g_{0}^{1-\theta} \\
& \quad \leq\left(\sum_{n \in \mathbf{Z}}\left|\varphi_{n, 0}\right| g_{0}\right)^{1-\theta}\left(\sum_{n \in \mathbf{Z}}\left|\varphi_{n, 0}\right|\right)^{\theta} \leq C\left(\sum_{n \in \mathbf{Z}}\left|\varphi_{n, 0}\right| g_{0}\right)^{1-\theta} .
\end{aligned}
$$

We then deduce that $\sum_{n \in \mathbf{Z}} \varphi_{n, 0} f_{0}$ converges to $f_{0}$ in $L^{p_{0} /(1-\theta)}\left(E_{0}^{(1 /(1-\theta))} ; w_{0}\right)$. Similarly, $\sum_{n \in \mathbf{Z}} \varphi_{n, 1} f_{1}$ converges to $f_{1}$ in $L^{p_{1} / \theta}\left(E_{1}^{(1 / \theta)} ; w_{1}\right)$. Now since one of $E_{0}$ and $E_{1}$ is $\sigma$-order continuous,

$$
\left(E_{0}, E_{1}\right)_{\theta}=E_{0}^{1-\theta} E_{1}^{\theta}=E_{0}^{(1 /(1-\theta))} E_{1}^{(1 / \theta)}
$$

(cf. [KPS, p. 244]. Note that by $E_{0}^{1-\theta} E_{1}^{\theta}$ we denote the quasi-Banach lattice introduced by Calderón [C]. The product of the lattices $E_{0}^{(1 /(1-\theta))}$ and $E_{1}^{(1 / \theta)}$ is defined in the natural way, see the next section). It then follows that

$$
\sum_{n \in \mathbf{Z}} \sum_{m \in \mathbf{Z}} \varphi_{n, 0} \varphi_{m, 1} f_{0} f_{1}
$$

converges to $f=f_{0} f_{1}$ in $L^{p}(E ; w)$. So there exist $n_{j}$ and $m_{j} \quad(j=0,1)$ such that

$$
\left\|f-\sum_{n=n_{0}}^{n_{1}} \varphi_{n, 0} f_{0} \sum_{m=m_{0}}^{m_{1}} \varphi_{m, 1} f_{1}\right\|_{H^{p}(E ; w)} \leq \varepsilon .
$$

Then we finish the proof of Theorem 6.3 as in $\S 4$. The remainder is omitted.

The following immediate consequence of Theorem 6.3 answers Problem 5 of [X1]. 
Corollary 6.4. Let $E$ be a quasi-Banach lattice of measurable functions on $(\Omega, \mu)$ such that $E^{(\alpha)}$ is UMD for some $\alpha>0$. Let $0<p_{0}, p_{1}<\infty$ and $0<\theta<1$. Then

$$
\left(H^{p_{0}}(E), H^{p_{1}}\left(L^{\infty}(\Omega)\right)\right)_{\theta}=H^{p}\left(\left(E, L^{\infty}(\Omega)\right)_{\theta}\right),
$$

with $\frac{1}{p}=\frac{1-\theta}{p_{0}}+\frac{\theta}{p_{1}}$.

Remarks. (i) It is evident that $\left(E, L^{\infty}(\Omega)\right)_{\theta}=E^{(1 /(1-\theta))}$.

(ii) Under the hypotheses of Corollary 6.4, (6.9) is also true for $p_{1}=\infty$ (see the remark after Lemma 6.2 and the arguments in §4). But at the time of this writing we do not know whether (6.8) and (6.9) hold if $p_{0}=p_{1}=\infty$.

\section{THE COUPLE $\left(H^{\infty}\left(E_{0}\right), H^{\infty}\left(E_{1}\right)\right)$}

The purpose of this section is to prove the following limit case of Theorem 5.3. Let $E$ be a Banach lattice of measurable functions on $(\Omega, \mu)$. We denote by $E^{\prime}$ the subspace of $E^{*}$ consisting of all the integrals.

Theorem 7.1. Let $E_{0}, E_{1}$ be quasi-Banach lattices of measurable functions on $(\Omega, \mu)$. Suppose that $E_{1}$ has $p$-majoration property $(0<p<\infty)$ and $E_{0}^{(\alpha)}$ is a UMD-space and $\left(E_{1}^{(\alpha)}\right)^{\prime}$ is a norming subspace for some $\alpha>0$. Then there exists a constant $C$ depending on $E_{0}$ and $E_{1}$ such that for any $t>0$ and any $f \in H^{\infty}\left(E_{0}\right)+H^{\infty}\left(E_{1}\right)$,

$$
K\left(t, f ; H^{\infty}\left(E_{0}\right), H^{\infty}\left(E_{1}\right)\right) \leq C K\left(t, f ; L^{\infty}\left(E_{0}\right), L^{\infty}\left(E_{1}\right)\right) .
$$

The proof of Theorem 7.1 is similar to and simplifies that of [X1, Proposition 6]. As in [X1], Theorem 7.1, will be derived from the following result, which seems to be of general interest.

Theorem 7.2. Let $E_{0}, E_{1}$ be quasi-Banach lattices of measurable functions on $(\Omega, \mu)$. Suppose that for some $\alpha_{0}>0$ and every $\alpha \geq \alpha_{0}$ there exists a constant $C=C\left(\alpha, E_{0}, E_{1}\right)$ such that

$$
\left\{\begin{array}{l}
\forall t>0, \forall f \in H^{\infty}\left(E_{0}^{(\alpha)}\right)+H^{\infty}\left(E_{1}^{(\alpha)}\right), \\
K\left(t, f ; H^{\infty}\left(E_{0}^{(\alpha)}\right), H^{\infty}\left(E_{1}^{(\alpha)}\right)\right) \leq C K\left(t, f ; L^{\infty}\left(E_{0}^{(\alpha)}\right), L^{\infty}\left(E_{1}^{(\alpha)}\right)\right) .
\end{array}\right.
$$

Then (7.2) is true for all $\alpha<\alpha_{0}$ as well (of course, with another constant $C$ ).

We remark at once that the method of proving Theorem 7.2 employed here is close to certain considerations of Pisier [P2]. The main idea of this proof has been discovered by the second-named author independently of [P2]. Note, by the way, that Pisier's method can be employed to give alternative proofs of Theorems 5.3 and 5.4 in most cases, but the entire development of this paper was independent of [P2].

Assuming Theorem 7.2 established, we can easily deduce Theorem 7.1. By our general hypotheses on lattices of measurable functions (see $\S 1$ ), there is $\alpha_{0}>0$ such that $E_{0}^{(\alpha)}$ and $E_{1}^{(\alpha)}$ are Banach lattices for $\alpha \geq \alpha_{0}$. We also choose $\alpha_{0}$ so large that $E_{0}^{\left(\alpha_{0}\right)}$ is UMD and $\left(E_{1}^{\left(\alpha_{0}\right)}\right)^{\prime}$ is norming. On the other hand, $E_{0}^{(\alpha)}$ and $E_{1}^{(\alpha)}$ still satisfy the hypotheses of Theorem 7.1 for $\alpha \geq \alpha_{0}$. 
Therefore, by Theorem 5.3, there exists a constant $C=C\left(\alpha, E_{0}, E_{1}\right)$ such that

$$
\left\{\begin{array}{l}
\forall t>0, \forall f \in H^{1}\left(E_{0}^{(\alpha)}\right)+H^{1}\left(E_{1}^{(\alpha)}\right), \\
K\left(t, f ; H^{1}\left(E_{0}^{(\alpha)}\right), H^{1}\left(E_{1}^{(\alpha)}\right)\right) \leq C K\left(t, f ; L^{1}\left(E_{0}^{(\alpha)}\right), L^{1}\left(E_{1}^{(\alpha)}\right)\right) .
\end{array}\right.
$$

As in [HP, Theorem 2.7] we dualize this statement but with $\left(E_{0}^{(\alpha)}\right)^{\prime}$ and $\left(E_{1}^{(\alpha)}\right)^{\prime}$ instead of their duals (this is possible because there spaces are norming); so we obtain the following $J$-functional estimate about the quotient spaces:

$$
\forall t>0, \forall \tilde{f} \in \frac{L^{\infty}\left(\left(E_{0}^{(\alpha)}\right)^{\prime}\right)}{H^{\infty}\left(\left(E_{0}^{(\alpha)}\right)^{\prime}\right)} \cap \frac{L^{\infty}\left(\left(E_{1}^{(\alpha)}\right)^{\prime}\right)}{H^{\infty}\left(\left(E_{1}^{(\alpha)}\right)^{\prime}\right)}
$$

there exists $f \in L^{\infty}\left(\left(E_{0}^{(\alpha)}\right)^{\prime}\right) \cap L^{\infty}\left(\left(E_{1}^{(\alpha)}\right)^{\prime}\right)$ representing $\tilde{f}$ in both quotient spaces and satisfying

$$
\begin{aligned}
& J\left(t, f ; L^{\infty}\left(\left(E_{0}^{(\alpha)}\right)^{\prime}\right), L^{\infty}\left(\left(E_{1}^{(\alpha)}\right)^{\prime}\right)\right) \\
& \quad \leq C J\left(t, \tilde{f} ; \frac{L^{\infty}\left(\left(E_{0}^{(\alpha)}\right)^{\prime}\right)}{H^{\infty}\left(\left(E_{0}^{(\alpha)}\right)^{\prime}\right)}, \frac{L^{\infty}\left(\left(E_{1}^{(\alpha)}\right)^{\prime}\right)}{H^{\infty}\left(\left(E_{1}^{(\alpha)}\right)^{\prime}\right)}\right) .
\end{aligned}
$$

Then using a simple factorization again as in [HP, Theorem 2.7], we see that this $J$-functional estimate is still true with $L^{\infty}, H^{\infty}$ replaced respectively by $L^{1}, H^{1}$. We dualize once more this last $J$-functional estimate to get (7.2). Thus (7.2) is true for every $\alpha \geq \alpha_{0}$, and it remains to apply Theorem 7.2.

We now proceed to the proof of Theorem 7.2. We first introduce some elementary notions. For two quasi-Banach lattices $E_{0}$ and $E_{1}$ of measurable functions on $(\Omega, \mu)$, let

$$
E_{0} E_{1}=\left\{x_{0} x_{1}: x_{j} \in E_{j}, j=0,1\right\}
$$

and for $x \in E_{0} E_{1}$

$$
\|x\|_{E_{0} E_{1}}=\inf \left\{\left\|x_{0}\right\|_{E_{0}}\left\|x_{1}\right\|_{E_{1}}: x=x_{0} x_{1}, x_{j} \in E_{j}, j=0,1\right\} .
$$

Then equipped with the above quasi-norm, $E_{0} E_{1}$ becomes a quasi-Banach lattice on $(\Omega, \mu)$. Similarly

$$
H^{\infty}\left(E_{0}\right) H^{\infty}\left(E_{1}\right)=\left\{f_{0} f_{1}: f_{j} \in H^{\infty}\left(E_{j}\right), j=0,1\right\} .
$$

Equipped with its natural quasi-norm, $H^{\infty}\left(E_{0}\right) H^{\infty}\left(E_{1}\right)$ is a quasi-Banach space. Using the construction of outer functions, it is easy to see that

$$
H^{\infty}\left(E_{0}\right) H^{\infty}\left(E_{1}\right)=H^{\infty}\left(E_{0} E_{1}\right) \quad \text { (with equality of quasi-norms). }
$$

Lemma 7.3. Let (7.2) be true for all $\alpha \geq 3 / 2$. Then

$$
H^{\infty}\left(E_{0}^{(2)} E_{1}^{(2)}\right) \subset\left(H^{\infty}\left(E_{0}\right), H^{\infty}\left(E_{1}\right)\right)_{\frac{1}{2} \infty} .
$$

Postponing the proof of Lemma 7.3 we are now ready to finish the proof of Theorem 7.2.

Proof of Theorem 7.2. Evidently, it suffices to show (7.2) for $\alpha=1$ supposing $\alpha_{0}=3 / 2$ (then we can iterate the procedure to gain smaller values of $\alpha$ ). So assume (7.2) is true for all $\alpha \geq 3 / 2$. Take $f \in H^{\infty}\left(E_{0}\right)+H^{\infty}\left(E_{1}\right)$ such that $K\left(t, f ; L^{\infty}\left(E_{0}\right), L^{\infty}\left(E_{1}\right)\right)<1$. Let $\Omega_{0}=\{\omega \in \Omega$ : the function $\zeta \mapsto f(\zeta, \omega)$ 
is not identically zero on $\mathbf{T}\}$. Since for some small $s>0 f(\cdot, \omega) \in H^{s}$ for a.e. $\omega \in \Omega$, we can write $f=u F$, where $u$ and $F$ are measurable functions on $\mathbf{T} \times \Omega$, analytic in the first variable, $u=F=0$ on $\mathbf{T} \times\left(\Omega \backslash \Omega_{0}\right), u(\cdot, \omega)$ inner and $F(\cdot, \omega)$ outer for a.e. $\omega \in \Omega_{0}$. In particular, $|f|=|F|$ a.e. on $\mathbf{T} \times \Omega$, and consequently

$$
K\left(t, F ; L^{\infty}\left(E_{0}\right), L^{\infty}\left(E_{1}\right)\right)<1,
$$

which yields

$$
K\left(t^{1 / 2}, F^{1 / 2} ; L^{\infty}\left(E_{0}^{(2)}\right), L^{\infty}\left(E_{1}^{(2)}\right)\right)<\sqrt{2} .
$$

Now $F^{1 / 2}$ is analytic in the first variable and so $F^{1 / 2} \in H^{\infty}\left(E_{0}^{(2)}\right)+H^{\infty}\left(E_{1}^{(2)}\right)$. Hence by (7.2) with $\alpha=2 \geq 3 / 2$

$$
K\left(t^{1 / 2}, F^{1 / 2} ; H^{\infty}\left(E_{0}^{(2)}\right), H^{\infty}\left(E_{1}^{(2)}\right)\right) \leq C .
$$

It then follows that there exists $F_{j} \in H^{\infty}\left(E_{j}^{(2)}\right)(j=0,1)$ such that

$$
F^{1 / 2}=F_{0}+F_{1} \text { and }\left\|F_{0}\right\|_{H^{\infty}\left(E_{0}^{(2)}\right)}+t^{1 / 2}\left\|F_{1}\right\|_{H^{\infty}\left(E_{1}^{(2)}\right)} \leq C \text {. }
$$

Then $f=u F=u F_{0}^{2}+u F_{1}^{2}+2 u F_{0} F_{1}$ and by (7.5), (7.4)

$$
\begin{aligned}
K(t, & \left.f H^{\infty}\left(E_{0}\right), H^{\infty}\left(E_{1}\right)\right) \\
\leq & C K\left(t, u F_{0}^{2}+u F_{1}^{2} ; H^{\infty}\left(E_{0}\right), H^{\infty}\left(E_{1}\right)\right) \\
& +C K\left(t, 2 u F_{0} F_{1} ; H^{\infty}\left(E_{0}\right), H^{\infty}\left(E_{1}\right)\right) \\
\leq & C+C K\left(t, u F_{0} F_{1} ; H^{\infty}\left(E_{0}\right), H^{\infty}\left(E_{1}\right)\right) \\
& \leq C+C t^{1 / 2}\left\|F_{0} F_{1}\right\|_{H^{\infty}\left(E_{0}^{(2)} E_{1}^{(2)}\right)} \leq C .
\end{aligned}
$$

This shows (7.2) for $\alpha=1$ by homogeneity and thus concludes the proof of Theorem 7.2.

Proof of Lemma 7.3. We claim first that given $r$ and $s$ with $1<r<s \leq 3$, we have

$$
H^{\infty}\left(E_{0}^{(r)} E_{1}^{\left(r^{\prime}\right)}\right) \subset\left(H^{\infty}\left(E_{0}\right), H^{\infty}\left(E_{0}^{(s)} E_{1}^{\left(s^{\prime}\right)}\right)\right)_{\theta_{0} \infty},
$$

where $\theta_{0}=s^{\prime} / r^{\prime}$ (for $1 \leq p \leq \infty, p^{\prime}$ stands for the conjugate exponent: $\left.1 / p+1 / p^{\prime}=1\right)$. Indeed, let $q$ be defined by $1 / r=1 / s+1 / q$. Then by (7.3)

$$
H^{\infty}\left(E_{0}^{(r)} E_{1}^{\left(r^{\prime}\right)}\right)=H^{\infty}\left(E_{0}^{(s)}\right) H^{\infty}\left(E_{0}^{(q)} E_{1}^{\left(r^{\prime}\right)}\right) .
$$

Now $1 / q+1 / r^{\prime}=1 / s^{\prime}$ and $s^{\prime} \geq 3 / 2$. Applying (7.2) with $\alpha=s^{\prime}$ and using interpolation result on vector-valued $L^{\infty}$-spaces, we obtain

$$
\left(H^{\infty}\left(E_{0}^{\left(s^{\prime}\right)}\right), H^{\infty}\left(E_{1}^{\left(s^{\prime}\right)}\right)\right)_{\theta_{0} \infty}=H^{\infty}\left(\left(E_{0}^{\left(s^{\prime}\right)}, E_{1}^{\left(s^{\prime}\right)}\right)_{\theta_{0} \infty}\right) .
$$

On the other hand, it is easy to check that

$$
E_{0}^{\left(s^{\prime} /\left(1-\theta_{0}\right)\right)} E_{1}^{\left(s^{\prime} / \theta_{0}\right)} \subset\left(E_{0}^{\left(s^{\prime}\right)}, E_{1}^{\left(s^{\prime}\right)}\right)_{\theta_{0} \infty} .
$$

Indeed, if $E_{0}$ and $E_{1}$ are Banach lattices, this is a consequence of the result (which is well known and easy to prove) on the relation between the real and complex interpolations (cf., e.g., [BL]). For quasi-Banach lattices considered in this paper there exists $\alpha>0$ such that $E_{0}^{(\alpha)}$ and $E_{1}^{(\alpha)}$ are equivalent to Banach lattices; so (7.8) is true for $E_{j}$ replaced by $E_{j}^{(\alpha)}(j=0,1)$, from which we easily deduce (7.8). 
By (7.7) and (7.8)

$$
H^{\infty}\left(E_{0}^{(q)} E_{1}^{\left(r^{\prime}\right)}\right) \subset\left(H^{\infty}\left(E_{0}^{\left(s^{\prime}\right)}\right), H^{\infty}\left(E_{1}^{\left(s^{\prime}\right)}\right)\right)_{\theta_{0} \infty}
$$

Multiplying this last inclusion by $H^{\infty}\left(E_{0}^{(s)}\right)$, we obtain (7.6).

Now applying (7.6) with $r=2$ and $s=3$, we obtain

$$
H^{\infty}\left(E_{0}^{(2)} E_{1}^{(2)}\right) \subset\left(H^{\infty}\left(E_{0}\right), H^{\infty}\left(E_{0}^{(3)} E_{1}^{(3 / 2)}\right)\right)_{\frac{3}{4} \infty} .
$$

Then interchanging the roles of the indices and taking $r=3 / 2$ and $s=2$ in (7.6), we have

$$
H^{\infty}\left(E_{1}^{(3 / 2)} E_{0}^{(3)}\right) \subset\left(H^{\infty}\left(E_{1}\right), H^{\infty}\left(E_{1}^{(2)} E_{0}^{(2)}\right)\right)_{\frac{2}{3} \infty}
$$

These two inclusions allow us to finish the proof of the lemma by a simple iteration procedure. Let $t^{1 / 2} H^{\infty}\left(E_{0}\right)+t^{-1 / 2} H^{\infty}\left(E_{1}\right)$ be $H^{\infty}\left(E_{0}\right)+H^{\infty}\left(E_{1}\right)$ equipped with the quasi-norm $t^{1 / 2} K\left(t, \cdot ; H^{\infty}\left(E_{0}\right), H^{\infty}\left(E_{1}\right)\right)$. To prove (7.4) is equivalent to prove that $H^{\infty}\left(E_{0}^{(2)} E_{1}^{(2)}\right)$ is included in $t^{1 / 2} H^{\infty}\left(E_{0}\right)+t^{-1 / 2} H^{\infty}\left(E_{1}\right)$ uniformly in $t>0$ (i.e., of inclusion norm dominated by a constant independent of $t$ ). To show this latter statement, fix $t>0$ and let $f \in H^{\infty}\left(E_{0}^{(2)} E_{1}^{(2)}\right)$ be of quasi-norm less than 1 . We shall construct a function $g \in H^{\infty}\left(E_{0}\right)+H^{\infty}\left(E_{1}\right)$ such that

$$
\|f-g\|_{H^{\infty}\left(E_{0}^{(2)} E_{1}^{(2)}\right)} \leq \varepsilon \text { and } t^{1 / 2} K\left(t, g ; H^{\infty}\left(E_{0}\right), H^{\infty}\left(E_{1}\right)\right) \leq C
$$

with a constant $C$ independent of $t$ and $f$ ( $\varepsilon$ being fixed and very small). An easy iteration will show that $H^{\infty}\left(E_{0}^{(2)} E_{1}^{(2)}\right)$ is included in $t^{1 / 2} H^{\infty}\left(E_{0}\right)+$ $t^{-1 / 2} H^{\infty}\left(E_{1}\right)$ of inclusion norm $\leq C$.

Let $C_{1}$ be a number bigger than the maximum of the inclusion norms of (7.9) and (7.10). Let $t_{1}>0$ and $t_{2}>0$ be two positive numbers. Then using consecutively (7.9) and (7.10), we get $f_{0}, f_{1}$ and $f^{\prime}$ such that $f=f_{0}+f_{1}+f^{\prime}$ and

$$
\begin{gathered}
\left\|f_{0}\right\|_{H^{\infty}\left(E_{0}\right)} \leq C_{1} t_{1}^{3 / 4}, \quad\left\|f_{1}\right\|_{H^{\infty}\left(E_{1}\right)} \leq C_{1}^{2} t_{1}^{-1 / 4} t_{2}^{2 / 3} \\
\left\|f^{\prime}\right\|_{H^{\infty}\left(E_{0}^{(2)} E_{1}^{(2)}\right)} \leq C_{1}^{2} t_{1}^{-1 / 4} t_{2}^{-1 / 3}
\end{gathered}
$$

Now choose $t_{1}=\delta^{2} t^{2 / 3}, t_{2}=\delta^{3 / 2} t^{-1 / 2}$ with $\delta>0$. Then

$$
\begin{gathered}
\left\|f_{0}\right\|_{H^{\infty}\left(E_{0}\right)} \leq C_{1} \delta^{3 / 2} t^{1 / 2}, \quad\left\|f_{1}\right\|_{H^{\infty}\left(E_{1}\right)} \leq C_{1}^{2} \delta^{1 / 2} t^{-1 / 2} \\
\left\|f^{\prime}\right\|_{H^{\infty}\left(E_{0}^{(2)} E_{1}^{(2)}\right)} \leq C_{1}^{2} \delta^{-1}
\end{gathered}
$$

Let $\delta=\varepsilon^{-1} C_{1}^{2}$ and $g=f_{0}+f_{1}$. Then $g \in H^{\infty}\left(E_{0}\right)+H^{\infty}\left(E_{1}\right)$, and the above inequalities give (7.11). We thus complete the proof of Lemma 7.3.

Remark. As pointed out after Theorem 7.2, some ideas of the preceding reasoning were also used in [P2]. More precisely, these are the deduction of (7.2) 
for $\alpha=1$ from (7.4) and (7.2) supposed true for $\alpha=2$, i.e., what is called in [P2] for "square argument", and that of (7.6) from (7.2) supposed true for $\alpha \geq 3 / 2$.

Remark. By the same argument as above we can show the following: Let $E_{0}, E_{1}$ be quasi-Banach lattices. Let $\alpha_{0}$ and $p_{0}>0$ be such that for every $\alpha, \beta \geq \alpha_{0}$ and $p, q \geq p_{0}$ there exists $C>0$ such that

$$
\left\{\begin{array}{l}
\forall t>0, \forall f \in H^{p}\left(E_{0}^{(\alpha)}\right)+H^{q}\left(E_{1}^{(\beta)}\right) \\
K\left(t, f ; H^{p}\left(E_{0}^{(\alpha)}\right), H^{q}\left(E_{1}^{(\beta)}\right)\right) \leq C K\left(t, f ; L^{p}\left(E_{0}^{(\alpha)}\right), L^{q}\left(E_{1}^{(\beta)}\right)\right) .
\end{array}\right.
$$

Then (7.12) holds for all $\alpha, \beta, p, q$.

This remark allows us to prove the following complement to Theorem 5.3.

Proposition 7.4. Let $\alpha>0$ and $E_{0}, E_{1}$ be quasi-Banach lattices of measurable functions on $(\Omega, \mu)$. Suppose that $E_{0}^{(\alpha)}, E_{1}^{(\alpha)}$ are Banach lattices either whose duals are Banach lattices satisfying the hypotheses of Theorem 5.3 or which are duals of two Banach lattices $F_{0}, F_{1}$ satisfying these hypotheses. Then for $0<p_{0}, p_{1} \leq \infty$ we have

$$
\left\{\begin{array}{l}
\forall t>0, \forall f \in H^{p_{0}}\left(E_{0}\right)+H^{p_{1}}\left(E_{1}\right), \\
K\left(t, f ; H^{p_{0}}\left(E_{0}\right), H^{p_{1}}\left(E_{1}\right)\right) \leq C K\left(t, f ; L^{p_{0}}\left(E_{0}\right), L^{p_{1}}\left(E_{1}\right)\right) .
\end{array}\right.
$$

where $C$ is a constant independent of $t$ and $f$.

Proof. For example, suppose the duals $\left(E_{0}^{(\alpha)}\right)^{*}$ and $\left(E_{1}^{(\alpha)}\right)^{*}$ satisfy the hypotheses of Theorem 5.3. Then for any $0<p_{0}, p_{1}<\infty,(5.5)$ holds with $E_{j}$ replaced by $\left(E_{j}^{(\alpha)}\right)^{*} \quad(j=0,1)$; so by the duality between $K$ - and $J$-functionals (cf. [BL]) we deduce (7.12) for all $1<p, q \leq \infty$ and $\alpha=\beta \geq \alpha_{0}$. Therefore, by the preceding remark, Proposition 7.4 is true in this case. We prove similarly the proposition in the remaining case.

A particular and interesting case of Proposition 7.4 is that when $E_{0}, E_{1}$ are Lorentz spaces on $(\Omega, \mu)$. Let

$$
E_{j}=L^{q_{j} r_{j}}(\Omega, \mu), \quad 0<q_{j}, r_{j} \leq \infty, j=0,1 .
$$

Then clearly, these spaces satisfy the hypotheses of Proposition 7.4. Therefore, we get

Corollary 7.5. Let $0<p_{j}, q_{j}, r_{j} \leq \infty \quad(j=0,1)$. Then there exists a constant $C$ depending on $0<p_{j}, q_{j}, r_{j} \leq \infty(j=0,1)$ such that for any $t>0$ and $f \in H^{p_{0}}\left(L^{q_{0} r_{0}}(\mu)\right)+H^{p_{1}}\left(L^{q_{1} r_{1}}(\mu)\right)$

$$
\begin{aligned}
K(t & \left., f ; H^{p_{0}}\left(L^{q_{0} r_{0}}(\mu)\right), H^{p_{1}}\left(L^{q_{1} r_{1}}(\mu)\right)\right) \\
& \leq C K\left(t, f ; L^{p_{0}}\left(L^{q_{0} r_{0}}(\mu)\right), L^{p_{1}}\left(L^{q_{1} r_{1}}(\mu)\right)\right) .
\end{aligned}
$$

\section{REFERENCES}

[AJ] K. F. Andersen and R. T. John, Weighted inequalities for vector-valued maximal functions and singular integrals, Studia Math. 69 (1980), 19-31.

[BL] J. Bergh and J. Löfström, Interpolation spaces. An introduction, Springer-Verlag, 1976.

[BX] O. Blasco and Q. Xu, Interpolation between vector-valued Hardy spaces, J. Funct. Anal. 102 (1991), 331-359. 
[B1] J. Bourgain, New Banach space properties of the disk algebra and $H^{\infty}$, Acta Math. 152 (1984), 1-46.

[B2] Bilinear forms on $H^{\infty}$ and bounded bianalytic functions, Trans. Amer. Math. Soc. 286 (1984), 313-338.

[B3] Some remarks on Banach spaces in which martingale differences are unconditional, Ark. Mat. 21 (1983), 163-168.

[Bu] D. L. Burkholder, Martingales and Fourier analysis in Banach spaces, Lecture Notes in Math., vol. 1206, Springer, 1983, pp. 61-108.

[C] A. P. Calderón, Intermediate spaces and interpolation: The complex method, Studia Math. 24 (1964), 113-190.

[CF] R. Coifman and C. Fefferman, Weighted norm inequalities for maximal functions and singular integrals, Studia Math. 51 (1974), 241-250.

[CMW] M. Cwikel, J. E. McCarthy, and T. H. Wolff, Interpolation between weighted Hardy spaces, Preprint 1990.

[CMS] M. Cwikel, M. Milman, and Y. Sagher, Complex interpolation of some quasi-Banach spaces, J. Funct. Anal. 65 (1986), 339-347.

[FRS] C. Fefferman, N. M. Riviere, and Y. Sagher, Interpolation between $H^{p}$ spaces: The real method, Trans. Amer. Math. Soc. 191 (1974), 71-81.

[GR] J. Garcia-Cuerva and J. L. Rubio de Francia, Weighted norm inequalities and related topics, North-Holland, Amsterdam, 1985.

[G] J. Garnett, Bounded analytic functions, Pure and Appl. Math., vol. 96, Academic Press, 1981.

[HP] U. Haagerup and G. Pisier, Factorization of analytic functions with values in non-commutative $L_{1}$-spaces and applications, Canad. J. Math. 41 (1989), 882-906.

[JJ] S. Janson and P. W. Jones, Interpolation between $H^{p}$-spaces: The complex method, J. Funct. Anal. 48 (1982), 58-80.

[J] P. W. Jones, Interpolation between Hardy spaces, Proc. Conf. on Harmonic Analysis in Honor of Antoni Zygmund, (Chicago, 1981), Belmont Wadsworth International, 1983.

[KA] L. V. Kantorovitsh and G. P. Akilov, Functional analysis, "Nauka", Moscow, 1977. (Russian)

[K1] S. V. Kisliakov, Truncating fucntions in weighted $H^{p}$ and two theorems of J. Bourgain, Preprint, Uppsala University, 1989.

[K2] Extension of $(q, p)$-summing operators defined on the disc-algebra with an appendix on Bourgain's analytic projection, Preprint 1990.

[K3] _ , $(q, p)$-summing operators on the disc algebra and a weighted estimate for certain outer functions, LOMI Leningrad, 1989, preprint E-11-89.

[K4] _ Absolutely summing operators on the disc algebra, Algebra and Analysis 3 (1991), no. 4; English transl. in St. Petersburg Math. J. 3 (1992).

[KPS] S. G. Krein, Ju. I. Petunin, and E. M. Semenov, Interpolation of linear operators, Trans. Math. Monos, vol. 54, Amer. Math., Soc., Providence, R.I., 1980.

[LT] J. Lindenstrauss and L. Tzafriri, Classical Banach spaces. II, Springer-Verlag, Berlin, 1979.

[P1] G. Pisier, A simple proof of a theorem of Jean Bourgain, Michigan J. Math. 39 (1992), 475-484.

[P2] - Interpolation between $H^{p}$ spaces and non-commutative generalizations. I, Pacific J. Math. 155 (1992), 341-368.

[RF] J. L. Rubio de Francia, Martingales and integral transforms of Banach space valued functions, Lecture Notes in Math., Springer, vol. 1221, 1985, pp. 195-222.

[S] J. D. Stafney, The spectrum of an operator on an interpolation space, Trans. Amer. Math. Soc. 144 (1969), 333-349.

[T] A. Torchinsky, Real-variable methods in harmonic anaysis, Pure and Appl. Math., vol. 123, Academic Press, 1986. 
[X1] Q. Xu, Real interpolation of some Banach lattices valued Hardy spaces, Bull. Sci. Math. 116 (1992), 227-246.

[X2] _ Notes on interpolation of Hardy spaces, Ann. Inst. Fourier (Grenoble) 42 (1992), 875-889; Erratum 43 (1993), 469.

Steklov Mathematics Institute, St. Petersburg (POMI), 27, Fontanka, 191011 St. PeTERSBURG, RUSSIA

Current address: Institute of Mathematics, Polish Academy of Sciences, ul. Sniadeckich 8, s.p. 137, 00-950, Warszawa, Poland

E-mail address: skis@lomi.spb.su

Universite des Sciences et TechniQues de Lille Flandres ARtois, U.R.A. C.N.R.S. D751, U.F.R. de Mathématiques Pures et appliquées, 59655 Villeneuve d'Asce Cedex, France Current address: Université Paris 6, Equipe d'Analyse, Boîte 186, Tour 46-0; $4^{e}$ Etage, 4, Place Jussieu 75230 Paris Cedex 05, France

E-mail address: qxeccr.jussieu.fr 